\title{
Semisimple extensions of the Standard Model gauge algebra
}

\author{
B. C. Allanach $\odot^{*}$ \\ DAMTP, University of Cambridge, Wilberforce Road, Cambridge CB3 OWA, United Kingdom \\ Ben Gripaios $\oplus^{\dagger}$ and Joseph Tooby-Smith $\odot^{\ddagger}$ \\ Cavendish Laboratory, University of Cambridge, J.J. Thomson Avenue, \\ Cambridge CB3 OHE, United Kingdom
}

(Received 6 May 2021; accepted 13 July 2021; published 31 August 2021)

\begin{abstract}
We show how one may classify all semisimple algebras containing the $\mathfrak{g} \mathfrak{t}(3) \oplus \mathfrak{g} \mathfrak{t}(2) \oplus \mathfrak{t}(1)$ symmetry of the Standard Model and acting on some given matter sector, enabling theories beyond the Standard Model with unification (partial or total) of symmetries (gauge or global) to be cataloged. With just a single generation of Standard Model fermions plus a singlet neutrino, the only gauge symmetries correspond to the well-known algebras $\mathfrak{g} \mathfrak{i t}(5), \mathfrak{g} \mathfrak{v}(10)$, and $\mathfrak{g} \mathfrak{t}(4) \oplus \mathfrak{g} \mathfrak{t}(2) \oplus \mathfrak{g} \mathfrak{t}(2)$, but with two or more generations a limited number of exotic symmetries mixing flavor, color, and electroweak degrees of freedom become possible. We provide a complete catalog in the case of three generations or fewer and outline how our method generalizes to cases with additional matter.
\end{abstract}

DOI: 10.1103/PhysRevD.104.035035

\section{INTRODUCTION}

In searching for theories of physics beyond the Standard Model (SM), it is of interest to ask how the gauge Lie algebra $\mathfrak{s} \mathfrak{m}:=\mathfrak{g} \mathfrak{t}(3) \oplus \mathfrak{g} \mathfrak{t}(2) \oplus \mathfrak{t}(1)$ could be extended to a larger Lie algebra $\mathfrak{g} \supset \mathfrak{s m}$. To give a useful answer to this question requires us to make some plausible assumptions, not least because there are, a priori, infinitely many such algebras, and because the question is anyway meaningless if we do not specify how $\mathfrak{g}$ acts on the physical degrees of freedom.

The list of possible $\mathfrak{g}$ not only becomes finite, but it also can be determined explicitly with the help of a computer, once we specify that $\mathfrak{g}$ is semisimple, as is indicated by the fact that ratios of hypercharges are simple fractions and by the fact that gauge couplings appear to unify, and that $g$ acts by a unitary (respectively, orthogonal) representation on some given complex (respectively, real) matter fields, so as to preserve the kinetic terms in the Lagrangian density. (Strictly speaking, to get a finite list we must discard the largest summand of $\mathfrak{g}$ that acts trivially on the matter, which is anyway of no interest, and identify algebras that lead to equivalent physical theories, as we discuss below. Thus, for

\footnotetext{
*B.C.Allanach@damtp.cam.ac.uk †ripaios@hep.phy.cam.ac.uk *jss85@cam.ac.uk
}

Published by the American Physical Society under the terms of the Creative Commons Attribution 4.0 International license. Further distribution of this work must maintain attribution to the author(s) and the published article's title, journal citation, and DOI. Funded by SCOAP ${ }^{3}$. us, $\mathfrak{g}$ will act via faithful representation.) The list may be further curtailed by insisting that $\mathfrak{g}$ be free of local anomalies (global anomalies require us to specify the gauge group, in general, and will not concern us here) with respect to fermionic matter, so that it can be gauged. ${ }^{1}$ Such a list can serve as a vade mecum for model builders.

In this paper, we find all such $\mathfrak{g}$ in the case where the matter fields are taken to be the three generations of quarks and leptons of the SM along with three $\mathfrak{s m}$-singlet fermions (invoked to give neutrinos their observed masses), bringing the total number of Weyl fermions to 48 . A valid $\mathfrak{g}$ is then given by an anomaly-free semisimple algebra that contains $\mathfrak{s} \mathfrak{m}$ and is contained in $\mathfrak{g} \mathfrak{t}(48)$. Two such algebras will lead to physical theories that are equivalent if they are related by an inner automorphism of $\mathfrak{g} \mathfrak{u}(48)$, since such an automorphism can be effected by a linear change of variables of the fermion fields, which leaves the path integral invariant. They will also lead to equivalent theories if they are related by an outer automorphism of $\mathfrak{g}$, since applying such a transformation does not change the image of $\mathfrak{g}$ in $\mathfrak{g} \mathfrak{i t}(48)$.

Although we study just one example, the methods we use can be generalized at whim. For example, one could easily include the scalar Higgs fields of the SM [in which case one seeks all $\mathfrak{g}$ containing $\mathrm{sm}$ and contained in $\mathfrak{s} \mathfrak{t}(48) \oplus \mathfrak{s} \mathfrak{v}(4)]$ or indeed with $n$ additional fermions

\footnotetext{
${ }^{1}$ If we discard the requirement that $\mathfrak{g}$ be semisimple, the list becomes infinite, even if we add only a single anomaly-free $\mathfrak{t}(1)$, as Ref. [1] shows.
} 
and $m$ additional scalars [in which case the containing algebra is $\mathfrak{g} \mathfrak{t}(48+n) \oplus \mathfrak{g} \mathfrak{o}(4+m)]$. In this way, we end up with a procedure for finding unification symmetries that is so general that a critic might judge it as being worthless; we therefore feel a fitting name for it is floccinaucinihilipilification.

To illustrate the results we obtain, it is useful to begin with the simpler case with just a single generation of quarks

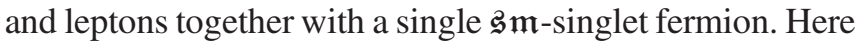
we already know that there are at least three possibilities, corresponding to the unification algebras $\mathfrak{p} \mathfrak{S}:=\mathfrak{g} \mathfrak{i t}(4) \oplus$

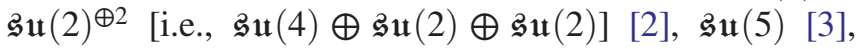
and $\mathfrak{s} \mathfrak{o}(10)$ [4,5] [which are all subalgebras of $\mathfrak{g} \mathfrak{t}(16)$; without the extra $\mathfrak{g m}$-singlet, we have just $\mathfrak{g} \mathfrak{t}(5) \subset$ $\mathfrak{g} \mathfrak{u}(15)]$; the "new result" with just a single generation, then, is that there are, perhaps unsurprisingly, no other possibilities. $^{2}$

Things become more interesting with more generations, because of the possibility of interplay between gauge and flavor symmetries. With two generations, for example, there are 45 possible algebras, up to equivalence. Some of these are easily guessed, such as the algebra $\mathfrak{g} \mathfrak{v}(10) \oplus \mathfrak{g} \mathfrak{t}(2)$, with the right-hand factor acting as a flavor symmetry mixing the two generations, along with $\mathfrak{S} \mathfrak{o}(10) \oplus \mathfrak{g} \mathfrak{i t}(5)$, with each summand acting nontrivially only on a single generation. But there also exist possibilities that are less easy to guess and which are interesting in that they mix up flavor and gauge symmetries in an essential way. One of these has algebra $\mathfrak{g} \mathfrak{t}(8) \oplus \mathfrak{g} \mathfrak{t}(2)^{\oplus 2}$, which generalizes with $N$ generations to $\mathfrak{g} \mathfrak{t}(4 N) \oplus \mathfrak{g} \mathfrak{u}(2)^{\oplus 2}$. This construction relies on the obvious embedding $\mathfrak{g} \mathfrak{t}(4) \oplus \mathfrak{s} \mathfrak{t}(N) \subset$ $\mathfrak{S} \mathfrak{t}(4 N)$, showing that it provides a generalization of the usual Pati-Salam symmetry $\mathfrak{p} \mathfrak{g}$ for $N=1$ containing an $\mathfrak{S} \mathfrak{u}(N)$ flavor symmetry. Thus, whereas in the usual Pati-Salam setup lepton number is interpreted as the fourth color, here flavor is to be interpreted as the remaining $4 N-4$ colors!

A qualitatively different generalization of the Pati-Salam model with two generations can be obtained as follows. Since the fermion fields in the one-generation version form two irreducible representations, there is a possible $\mathfrak{g} \mathfrak{t}(2)^{\oplus 2}$ flavor symmetry when we go to two generations, giving the algebra $\mathfrak{s} \mathfrak{t}(4) \oplus \mathfrak{g} \mathfrak{t}(2)^{\oplus 4}$, with the 32 fermion fields arranging themselves into the representation $(\mathbf{4}, \mathbf{2}, \mathbf{2}, \mathbf{1}, \mathbf{1}) \oplus(\overline{\mathbf{4}}, \mathbf{1}, \mathbf{1}, \mathbf{2}, \mathbf{2})$. This is anomaly free because $\mathfrak{g} \mathfrak{t}(2)$ has no anomalous representations. Noting that $\mathfrak{g} \mathfrak{t}(2)^{\oplus 2} \cong \mathfrak{S} \mathfrak{o}(4) \subset \mathfrak{S} \mathfrak{o}(5) \cong \mathfrak{s} \mathfrak{p}(4)$ and that the defining representation of $\mathfrak{s p}(4)$ restricts to the $(\mathbf{2 , 2 )}$ of

\footnotetext{
${ }^{2}$ In each case there is a single nontrivial outer automorphism of $\mathfrak{g}$, so without this equivalence we would find six possibilities, corresponding to the fact that one could assign the fermions to carry either of the inequivalent 16-dimensional spinor representations of $\mathfrak{g} \mathfrak{o}(10)$, say.
}

$\mathfrak{S} \mathfrak{t}(2)^{\oplus 2}$, it follows that this can be enlarged even further to $\mathfrak{s} \mathfrak{i t}(4) \oplus \mathfrak{s} \mathfrak{p}(4)^{\oplus 2}$, again leading to an essential mixing of flavor symmetry with $\mathfrak{s m}$.

Since this last construction relies on "accidental" isomorphisms of low-dimensional Lie algebras, we do not expect it to generalize to $N>2$ generations. Two qualitatively new algebras do appear, however. One uses the embeddings $\quad \mathfrak{g} \mathfrak{i t}(16 N) \supset \mathfrak{g} \mathfrak{t}(4) \oplus \mathfrak{I p}(2 N)^{\oplus 2} \subset \mathfrak{g} \mathfrak{t}(4) \oplus$ $(\mathfrak{g} \mathfrak{v}(N) \oplus \mathfrak{S p}(2))^{\oplus 2}$ along with the isomorphism $\mathfrak{s p}(2) \cong$ $\mathfrak{s} \mathfrak{u}(2)$ to produce an algebra containing $\mathfrak{p} \mathfrak{g}$ along with an $\mathfrak{g} \mathfrak{p}(N)^{\oplus 2}$ flavor symmetry. The other uses the embeddings $\quad \mathfrak{g} \mathfrak{t}(16 N) \supset \mathfrak{g} \mathfrak{t}(4) \oplus \mathfrak{a p}(2 N) \oplus \mathfrak{g} \mathfrak{v}(2 N) \subset \mathfrak{g} \mathfrak{t}(4) \oplus$ $\mathfrak{S} \mathfrak{v}(N) \oplus \mathfrak{S p}(2) \oplus \mathfrak{g} \mathfrak{v}(2) \oplus \mathfrak{S} \mathfrak{o}(N)$, to produce an algebra containing not $\mathfrak{p} \mathfrak{g}$, but rather its subalgebra $\mathfrak{g} \mathfrak{t}(4) \oplus$ $\mathfrak{S} \mathfrak{u}(2) \oplus \mathfrak{S} \mathfrak{o}(2) \supset \mathfrak{s} \mathfrak{m}$, which is not only not semisimple, but is also not left-right symmetric. Again we find a flavor symmetry isomorphic to $\mathfrak{s} \mathfrak{o}(N)^{\oplus 2}$, but now embedded differently in the SM flavor symmetry. These constructions rely on the embeddings $\mathfrak{s} \mathfrak{o}(2) \oplus \mathfrak{s} \mathfrak{o}(N) \subset \mathfrak{g} \mathfrak{o}(2 N)$ and $\mathfrak{s p}(2) \oplus \mathfrak{s} \mathfrak{p}(N) \subset \mathfrak{s} \mathfrak{p}(2 N)$. Note that in these examples, flavor symmetry is unified with the electroweak symmetry rather than with the strong symmetry, and in a variety of ways. ${ }^{3}$

The upshot is that with three generations we get many more algebras (340, up to equivalence) but all of them can be regarded as variations on the themes already described. This shows that the model building possibilities are in fact extremely limited, unless we include additional fermion fields. Nevertheless, we find a small number of interesting possibilities which mix gauge and flavor symmetries in an essential way. In particular, if such symmetries are gauged, the corresponding gauge bosons can change both flavor and color/electroweak charges of matter fields.

The algebras organize themselves into 26 (respectively, six) equivalence classes of semisimple anomaly-free algebras that are maximal (respectively, minimal) with respect to inclusion [note that $\mathfrak{g} \mathfrak{a t}(48)$ is not anomaly free, and $\mathfrak{g} \mathfrak{t}(3) \oplus \mathfrak{S} \mathfrak{t}(2) \oplus \mathfrak{t}(1)$ is not semisimple, so these definitions are cromulent]. We list these in Table I. The remaining algebras can be found in Table II. Additional information about the embeddings can be found in the Supplemental Matrials [6].

\section{THEORY}

We now describe the mathematical formulation of the problem. Because of the need to track automorphisms, this is most easily done using the language of category theory. A suitable category has objects, labeled $(\mathfrak{g}, \alpha, \beta, \gamma)$, given by commuting diagrams of the form

\footnotetext{
${ }^{3}$ For an even number of generations, we also have an embedding $\mathfrak{g} \mathfrak{t}(16 N) \supset \mathfrak{I} \mathfrak{t}(4) \oplus \mathfrak{I} \mathfrak{o}(2 N)^{\oplus 2} \supset \mathfrak{I} \mathfrak{t}(4) \oplus(\mathfrak{I p}(2) \oplus$ $\mathfrak{I p}(N))^{\oplus 2}$, using the embedding $\mathfrak{s p}(2) \oplus \mathfrak{s p}(N) \subset \mathfrak{s} \mathfrak{p}(2 N)$.
} 
TABLE I. All maximal and minimal anomaly-free algebras for exactly three generations of SM fermions plus three right-handed neutrinos.

\begin{tabular}{|c|c|c|}
\hline \multicolumn{3}{|r|}{ Maximal } \\
\hline & Algebra & Fermion representations corresponding to $\beta$ \\
\hline 1 & $\mathfrak{S} \mathfrak{o}(10) \oplus \mathfrak{g} \mathfrak{t}(2)$ & $(16,3)$ \\
\hline 2 & $\mathfrak{S o}(10)^{\oplus 3}$ & $(16,1,1) \oplus(1,16,1) \oplus(1,1,16)$ \\
\hline 3 & $\mathfrak{S} \mathfrak{o}(10)^{\oplus 2} \oplus \mathfrak{g} \mathfrak{u}(2)$ & $(16,1,1) \oplus(1,16,2)$ \\
\hline 4 & $\mathfrak{g} \mathfrak{u}(4) \oplus \mathfrak{s} \mathfrak{p}(6)^{\oplus 2}$ & $(\overline{4}, \mathbf{6}, \mathbf{1}) \oplus(\mathbf{4}, \mathbf{1}, \mathbf{6})$ \\
\hline 5 & $\mathfrak{S} \mathfrak{u}(4)^{\oplus 2} \oplus \mathfrak{s p}(6)$ & $(\overline{4}, 6,1) \oplus(4,1,6)$ \\
\hline 6 & $\mathfrak{S} \mathfrak{i t}(12) \oplus \mathfrak{S} \mathfrak{u}(2)^{\oplus 2}$ & $(\overline{12}, 2,1) \oplus(12,1,2)$ \\
\hline 7 & $\mathfrak{s} \mathfrak{t}(4) \oplus \mathfrak{s} \mathfrak{p}(4)^{\oplus 2} \oplus \mathfrak{s} \mathfrak{v}(10)$ & $(\overline{4}, 4,1,1) \oplus(4,1,4,1) \oplus(1,1,1,16)$ \\
\hline 8 & $\mathfrak{G} \mathfrak{i t}(5) \oplus \mathfrak{G} \mathfrak{t}(2)^{\oplus 3}$ & $(\overline{5}, 3,1,1) \oplus(10,1,3,1) \oplus(1,1,1,2) \oplus(1,1,1,1)$ \\
\hline 9 & $\mathfrak{S} \mathfrak{t}(5) \oplus \mathfrak{G} \mathfrak{t}(2)^{\oplus 3}$ & $(\overline{\mathbf{5}}, \mathbf{3}, \mathbf{1}, \mathbf{1}) \oplus(\mathbf{1 0}, \mathbf{1}, \mathbf{3}, \mathbf{1}) \oplus(\mathbf{1}, \mathbf{1}, \mathbf{1}, \mathbf{3})$ \\
\hline 10 & $\mathfrak{S} \mathfrak{i t}(5) \oplus \mathfrak{g} \mathfrak{t}(2)^{\oplus 3}$ & $(\overline{\mathbf{5}}, \mathbf{1}, \mathbf{1}, \mathbf{1}) \oplus(\overline{\mathbf{5}}, \mathbf{2}, \mathbf{1}, \mathbf{1}) \oplus(\mathbf{1 0}, \mathbf{1}, \mathbf{3}, \mathbf{1}) \oplus(\mathbf{1}, \mathbf{1}, \mathbf{1}, \mathbf{2}) \oplus(\mathbf{1}, \mathbf{1}, \mathbf{1}, \mathbf{1})$ \\
\hline 11 & $\mathfrak{S} \mathfrak{t}(5) \oplus \mathfrak{G} \mathfrak{t}(2)^{\oplus 3}$ & $(\overline{\mathbf{5}}, \mathbf{1}, \mathbf{1}, \mathbf{1}) \oplus(\overline{\mathbf{5}}, \mathbf{2}, \mathbf{1}, \mathbf{1}) \oplus(\mathbf{1 0}, \mathbf{1}, \mathbf{3}, \mathbf{1}) \oplus(\mathbf{1}, \mathbf{1}, \mathbf{1}, \mathbf{3})$ \\
\hline 12 & $\mathfrak{S} \mathfrak{u}(5) \oplus \mathfrak{g} \mathfrak{t}(2)^{\oplus 3}$ & $(10,1,1,1) \oplus(\overline{5}, 3,1,1) \oplus(10,1,2,1) \oplus(1,1,1,2) \oplus(1,1,1,1)$ \\
\hline 13 & $\mathfrak{S} \mathfrak{u}(5) \oplus \mathfrak{S} \mathfrak{t}(2) \oplus 3$ & $(10,1,1,1) \oplus(\overline{5}, 3,1,1) \oplus(10,1,2,1) \oplus(1,1,1,3)$ \\
\hline 14 & $\mathfrak{G} \mathfrak{i t}(5)^{\oplus 2} \oplus \mathfrak{S} \mathfrak{o}(10) \oplus \mathfrak{G} \mathfrak{t}(2)$ & $(\overline{5}, 1,1,1) \oplus(10,1,1,1) \oplus(1, \overline{5}, 1,1) \oplus(1,10,1,1) \oplus(1,1,16,1) \oplus(1,1,1,2)$ \\
\hline 15 & $\mathfrak{G} \mathfrak{t}(5)^{\oplus 3} \oplus \mathfrak{S} \mathfrak{t}(2)$ & $\begin{array}{l}(\overline{\mathbf{5}}, \mathbf{1}, \mathbf{1}, \mathbf{1}) \oplus(\mathbf{1 0}, \mathbf{1}, \mathbf{1}, \mathbf{1}) \oplus(\mathbf{1}, \overline{\mathbf{5}}, \mathbf{1}, \mathbf{1}) \oplus(\mathbf{1}, \mathbf{1 0}, \mathbf{1}, \mathbf{1}) \oplus(\mathbf{1}, \mathbf{1}, \overline{\mathbf{5}}, \mathbf{1}) \oplus(\mathbf{1}, \mathbf{1}, \mathbf{1 0}, \mathbf{1}) \oplus \\
\quad(\mathbf{1}, \mathbf{1}, \mathbf{1}, \mathbf{3})\end{array}$ \\
\hline 16 & $\mathfrak{G} \mathfrak{t}(8) \oplus \mathfrak{S} \mathfrak{v}(10) \oplus \mathfrak{G} \mathfrak{t}(2)^{\oplus 2}$ & $(\mathbf{1}, \mathbf{1 6}, \mathbf{1}, \mathbf{1}) \oplus(\overline{\mathbf{8}}, \mathbf{1}, \mathbf{2}, \mathbf{1}) \oplus(\mathbf{8}, \mathbf{1}, \mathbf{1}, \mathbf{2})$ \\
\hline 17 & $\mathfrak{S} \mathfrak{u}(4) \oplus \mathfrak{g} \mathfrak{p}(4) \oplus \mathfrak{G} \mathfrak{o}(10) \oplus \mathfrak{G} \mathfrak{u}(2)^{\oplus 2}$ & $(\overline{4}, 4,1,1,1) \oplus(1,1,16,1,1) \oplus(4,1,1,2,2)$ \\
\hline 18 & $\mathfrak{S} \mathfrak{t}(4) \oplus \mathfrak{g} \mathfrak{p}(4) \oplus \mathfrak{g} \mathfrak{o}(10) \oplus \mathfrak{G} \mathfrak{u}(2)^{\oplus 2}$ & $(\overline{4}, 4,1,1,1) \oplus(1,1,16,1,1) \oplus(4,1,1,2,2)$ \\
\hline 19 & $\mathfrak{S} \mathfrak{i t}(4) \oplus \mathfrak{S p}(6) \oplus \mathfrak{S} \mathfrak{i t}(2)^{\oplus 3}$ & $(\overline{4}, 6,1,1,1) \oplus(4,1,2,2,1) \oplus(4,1,1,1,2)$ \\
\hline 20 & $\mathfrak{S} \mathfrak{i t}(4) \oplus \mathfrak{S} \mathfrak{p}(6) \oplus \mathfrak{S} \mathfrak{i t}(2)^{\oplus 3}$ & $(\overline{4}, 6,1,1,1) \oplus(4,1,2,2,1) \oplus(4,1,1,1,2)$ \\
\hline 21 & $\mathfrak{S} \mathfrak{t}(4)^{\oplus 2} \oplus \mathfrak{G} \mathfrak{t}(2)^{\oplus 3}$ & $(\overline{4}, 6,1,1,1) \oplus(4,1,2,2,1) \oplus(4,1,1,1,2)$ \\
\hline 22 & $\mathfrak{G} \mathfrak{u}(5) \oplus \mathfrak{S} \mathfrak{v}(10) \oplus \mathfrak{G} \mathfrak{u}(2)^{\oplus 3}$ & $(1,16,1,1,1) \oplus(\overline{5}, 1,2,1,1) \oplus(10,1,1,2,1) \oplus(1,1,1,1,2)$ \\
\hline 23 & $\mathfrak{G} \mathfrak{u}(5)^{\oplus 2} \oplus \mathfrak{S} \mathfrak{t}(2)^{\oplus 3}$ & $\begin{array}{l}(\mathbf{1}, \overline{\mathbf{5}}, \mathbf{1}, \mathbf{1}, \mathbf{1}) \oplus(\mathbf{1}, \mathbf{1 0}, \mathbf{1}, \mathbf{1}, \mathbf{1}) \oplus(\overline{\mathbf{5}}, \mathbf{1}, \mathbf{2}, \mathbf{1}, \mathbf{1}) \oplus(\mathbf{1 0}, \mathbf{1}, \mathbf{1}, \mathbf{2}, \mathbf{1}) \oplus(\mathbf{1}, \mathbf{1}, \mathbf{1}, \mathbf{1}, \mathbf{2}) \oplus \\
\quad(\mathbf{1}, \mathbf{1}, \mathbf{1}, \mathbf{1}, \mathbf{1})\end{array}$ \\
\hline 24 & $\mathfrak{S} \mathfrak{t}(5)^{\oplus 2} \oplus \mathfrak{S} \mathfrak{u}(2)^{\oplus 3}$ & $(\mathbf{1}, \overline{\mathbf{5}}, \mathbf{1}, \mathbf{1}, \mathbf{1}) \oplus(\mathbf{1}, \mathbf{1 0}, \mathbf{1}, \mathbf{1}, \mathbf{1}) \oplus(\overline{\mathbf{5}}, \mathbf{1}, 2, \mathbf{1}, \mathbf{1}) \oplus(10,1,1,2,1) \oplus(1,1,1,1,3)$ \\
\hline 25 & $\mathfrak{S} \mathfrak{t}(4) \oplus \mathfrak{S} \mathfrak{v}(10) \oplus \mathfrak{G} \mathfrak{t}(2)^{\oplus 4}$ & $(1,16,1,1,1,1) \oplus(4,1,2,2,1,1) \oplus(\overline{4}, 1,1,1,2,2)$ \\
\hline 26 & $\mathfrak{G} \mathfrak{t}(4)^{\oplus 2} \oplus \mathfrak{S} \mathfrak{p}(4)^{\oplus 2} \oplus \mathfrak{S} \mathfrak{t}(2)^{\oplus 2}$ & $(\overline{4}, \mathbf{1}, \mathbf{4}, \mathbf{1}, \mathbf{1}, \mathbf{1}) \oplus(\mathbf{4}, \mathbf{1}, \mathbf{1}, \mathbf{4}, \mathbf{1}, \mathbf{1}) \oplus(\mathbf{1}, \overline{4}, \mathbf{1}, \mathbf{1}, \mathbf{2}, \mathbf{1}) \oplus(\mathbf{1}, \mathbf{4}, \mathbf{1}, \mathbf{1}, \mathbf{1}, 2)$ \\
\hline & & 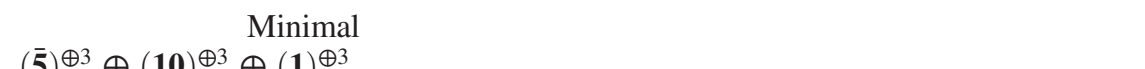 \\
\hline $\begin{array}{l}27 \\
28\end{array}$ & $\begin{array}{l}\mathfrak{S} \mathfrak{t}(5) \\
\mathfrak{G} \mathfrak{t}(4) \oplus \mathfrak{g} \mathfrak{t}(2)^{\oplus 2}\end{array}$ & $\begin{array}{l}(\mathbf{5})^{\oplus 3} \oplus(\mathbf{1 0})^{\oplus 3} \oplus(\mathbf{1})^{\oplus 3} \\
(\overline{\mathbf{4}}, \mathbf{2}, \mathbf{1})^{\oplus^{3}} \oplus(\mathbf{4}, \mathbf{1}, \mathbf{2})^{\oplus 3}\end{array}$ \\
\hline $\begin{array}{l}28 \\
29\end{array}$ & $\begin{array}{l}\mathfrak{S} \mathfrak{u}(4) \oplus \mathfrak{s} \mathfrak{u}(2) \\
\mathfrak{S} \mathfrak{t}(4)^{\oplus 2} \oplus \mathfrak{S} \mathfrak{t}(2)\end{array}$ & $(\overline{\mathbf{4}}, \mathbf{6}, \mathbf{1}) \oplus(\mathbf{4}, \mathbf{1}, \mathbf{2})^{\oplus 3}$ \\
\hline 30 & $\mathfrak{S} \mathfrak{u}(4) \oplus \mathfrak{S} \mathfrak{u}(5) \oplus \mathfrak{S} \mathfrak{u}(2) \oplus 2$ & $(\mathbf{1}, \overline{\mathbf{5}}, \mathbf{1}, \mathbf{1})^{\oplus 2} \oplus(\mathbf{1}, \mathbf{1 0}, \mathbf{1}, \mathbf{1})^{\oplus 2} \oplus(\overline{\mathbf{4}}, \mathbf{1}, \mathbf{2}, \mathbf{1}) \oplus(\mathbf{4}, \mathbf{1}, \mathbf{1}, \mathbf{2}) \oplus(\mathbf{1}, \mathbf{1}, \mathbf{1}, \mathbf{1})^{\oplus 2}$ \\
\hline 31 & $\mathfrak{S} \mathfrak{u}(4) \oplus \mathfrak{S} \mathfrak{u}(5) \oplus \mathfrak{S} \mathfrak{u}(2)^{\oplus 2}$ & $(\mathbf{1}, \overline{\mathbf{5}}, \mathbf{1}, \mathbf{1}) \oplus(\mathbf{1}, \mathbf{1 0}, \mathbf{1}, \mathbf{1}) \oplus(\overline{\mathbf{4}}, \mathbf{1}, \mathbf{2}, \mathbf{1})^{\oplus 2} \oplus(\mathbf{4}, \mathbf{1}, \mathbf{1}, \mathbf{2})^{\oplus 2} \oplus(\mathbf{1}, \mathbf{1}, \mathbf{1}, \mathbf{1})$ \\
\hline & $\mathfrak{S} \mathfrak{i t}(4)^{\oplus 3} \oplus \mathfrak{s} \mathfrak{t}(2)^{\oplus 2}$ & $\begin{array}{l}(\overline{4}, 1,1,2,1) \oplus(1,4,1,2,1) \oplus(1,1, \overline{4}, 2,1) \oplus(4,1,1,1,2) \oplus(1,4,1,1,2) \oplus \\
\quad(1,1,4,1,2)\end{array}$ \\
\hline
\end{tabular}

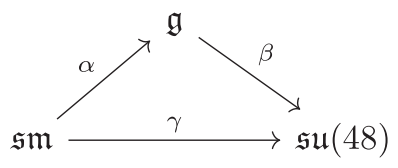

(1)

where $\mathfrak{g}$ is a semisimple Lie algebra, and $\alpha, \beta, \gamma$ are embeddings, i.e., injective maps that preserve Lie brackets. A morphism, labeled $(j, i)$, from $\left(\mathfrak{g}^{\prime}, \alpha^{\prime}, \beta^{\prime}, \gamma^{\prime}\right)$ to $(\mathfrak{g}, \alpha, \beta, \gamma)$ is then a commuting diagram of the form

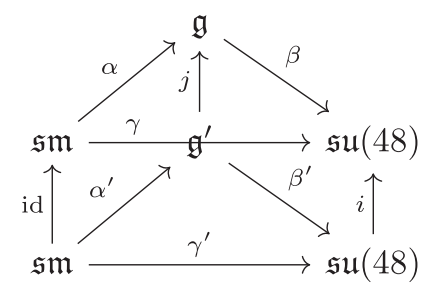

where $j$ is an embedding and $i$ is an inner automorphism. We call a morphism $(j, i)$ an equivalence if $j$ is an 
TABLE II. All algebras for exactly three generations of SM fermions plus three right-handed neutrinos which are neither maximal nor minimal.

\begin{tabular}{|c|c|c|}
\hline \multicolumn{3}{|r|}{ Nonmaximal and Nonminimal algebras } \\
\hline & Algebra & Fermion representations corresponding to $\beta$ \\
\hline 33 & $\mathfrak{S \mathfrak { o }}(10)$ & $(\mathbf{1 6})^{\oplus 3}$ \\
\hline 34 & $\mathfrak{s o}(10)^{\oplus 2}$ & $(16,1) \oplus(1,16)^{\oplus 2}$ \\
\hline 35 & $\mathfrak{S o}(10) \oplus \mathfrak{g} \mathfrak{t}(2)$ & $(16,1) \oplus(16,2)$ \\
\hline 36 & $\mathfrak{S} \mathfrak{t}(5) \oplus \mathfrak{S} \mathfrak{v}(10)$ & $(\overline{\mathbf{5}}, \mathbf{1}) \oplus(\mathbf{1 0}, \mathbf{1}) \oplus(\mathbf{1}, \mathbf{1 6})^{\oplus 2} \oplus(\mathbf{1}, \mathbf{1})$ \\
\hline 37 & $\mathfrak{G} \mathfrak{t}(5) \oplus \mathfrak{S} \mathfrak{o}(10)$ & $(\overline{\mathbf{5}}, \mathbf{1})^{\oplus 2} \oplus(\mathbf{1 0}, \mathbf{1})^{\oplus 2} \oplus(\mathbf{1}, \mathbf{1 6}) \oplus(\mathbf{1}, \mathbf{1})^{\oplus 2}$ \\
\hline 38 & $\mathfrak{G} \mathfrak{t}(5) \oplus \mathfrak{s} \mathfrak{u}(2)$ & $(\overline{\mathbf{5}}, \mathbf{3}) \oplus(\mathbf{1 0}, \mathbf{3}) \oplus(\mathbf{1}, \mathbf{1})^{\oplus 3}$ \\
\hline 39 & $\mathfrak{S} \mathfrak{u}(5) \oplus \mathfrak{G} \mathfrak{i t}(2)$ & $(\overline{\mathbf{5}}, \mathbf{1}) \oplus(\overline{\mathbf{5}}, \mathbf{2}) \oplus(\mathbf{1 0}, \mathbf{3}) \oplus(\mathbf{1}, \mathbf{1})^{\oplus 3}$ \\
\hline 40 & $\mathfrak{S} \mathfrak{u}(5) \oplus \mathfrak{S} \mathfrak{u}(2)$ & $(\overline{\mathbf{5}}, \mathbf{3}) \oplus(\mathbf{1 0}, \mathbf{3}) \oplus(\mathbf{1}, \mathbf{2}) \oplus(\mathbf{1}, \mathbf{1})$ \\
\hline 41 & $\mathfrak{G H} \mathfrak{t}(5) \oplus \mathfrak{G} \mathfrak{t}(2)$ & $(\overline{\mathbf{5}}, \mathbf{3}) \oplus(\mathbf{1 0}, \mathbf{3}) \oplus(\mathbf{1}, \mathbf{3})$ \\
\hline 42 & $\mathfrak{S} \mathfrak{t}(5) \oplus \mathfrak{G} \mathfrak{t}(2)$ & $(\mathbf{1 0}, \mathbf{1}) \oplus(\overline{\mathbf{5}}, \mathbf{3}) \oplus(\mathbf{1 0}, \mathbf{2}) \oplus(\mathbf{1 0}, \mathbf{1})^{\oplus 3}$ \\
\hline 43 & $\mathfrak{S} \mathfrak{u}(5) \oplus \mathfrak{G} \mathfrak{i t}(2)$ & $(\overline{\mathbf{5}}, \mathbf{1})^{\oplus 3} \oplus(\mathbf{1 0}, \mathbf{3}) \oplus(\mathbf{1}, \mathbf{1})^{\oplus 3}$ \\
\hline 44 & $\mathfrak{S} \mathfrak{1 t}(5) \oplus \mathfrak{S} \mathfrak{t}(2)$ & $(\overline{\mathbf{5}}, \mathbf{1}) \oplus(\overline{\mathbf{5}}, \mathbf{2}) \oplus(\mathbf{1 0}, \mathbf{3}) \oplus(\mathbf{1}, \mathbf{2}) \oplus(\mathbf{1}, \mathbf{1})$ \\
\hline 45 & $\mathfrak{G} \mathfrak{u}(5) \oplus \mathfrak{G} \mathfrak{t}(2)$ & $(\overline{\mathbf{5}}, \mathbf{1}) \oplus(\overline{\mathbf{5}}, \mathbf{2}) \oplus(\mathbf{1 0}, \mathbf{3}) \oplus(\mathbf{1}, \mathbf{3})$ \\
\hline 46 & $\mathfrak{G} \mathfrak{u}(5) \oplus \mathfrak{G} \mathfrak{i t}(2)$ & $(\overline{\mathbf{5}}, \mathbf{1}) \oplus(\mathbf{1 0}, \mathbf{1}) \oplus(\overline{\mathbf{5}}, \mathbf{2}) \oplus(\mathbf{1 0}, \mathbf{2}) \oplus(\mathbf{1}, \mathbf{1})^{\oplus 3}$ \\
\hline 47 & $\mathfrak{S} \mathfrak{i t}(5) \oplus \mathfrak{G} \mathfrak{i t}(2)$ & $(10,1) \oplus(\overline{5}, 3) \oplus(10,2) \oplus(1,2) \oplus(10,1)$ \\
\hline 48 & $\mathfrak{G} \mathfrak{u}(5) \oplus \mathfrak{G} \mathfrak{u}(2)$ & $(\mathbf{1 0}, \mathbf{1}) \oplus(\overline{\mathbf{5}}, \mathbf{3}) \oplus(\mathbf{1 0}, \mathbf{2}) \oplus(\mathbf{1}, \mathbf{3})$ \\
\hline 49 & $\mathfrak{S} \mathfrak{i t}(5) \oplus \mathfrak{S} \mathfrak{t}(2)$ & $(\mathbf{1 0}, \mathbf{1})^{\oplus 3} \oplus(\overline{\mathbf{5}}, \mathbf{3}) \oplus(\mathbf{1 0}, \mathbf{1})^{\oplus 3}$ \\
\hline 50 & $\mathfrak{S H} \mathfrak{t}(5) \oplus \mathfrak{S} \mathfrak{t}(2)$ & $(\overline{\mathbf{5}}, \mathbf{1})^{\oplus 3} \oplus(\mathbf{1 0}, \mathbf{1}) \oplus(\mathbf{1 0}, \mathbf{2}) \oplus(\mathbf{1}, \mathbf{1})^{\oplus 3}$ \\
\hline 51 & $\mathfrak{G} \mathfrak{u t}(5) \oplus \mathfrak{G} \mathfrak{i t}(2)$ & $(\overline{\mathbf{5}}, \mathbf{1})^{\oplus 3} \oplus(\mathbf{1 0}, \mathbf{3}) \oplus(\mathbf{1}, \mathbf{2}) \oplus(\mathbf{1}, \mathbf{1})$ \\
\hline 52 & $\mathfrak{S t \mathfrak { t }}(5) \oplus \mathfrak{S} \mathfrak{i t}(2)$ & $(\overline{\mathbf{5}}, \mathbf{1})^{\oplus 3} \oplus(\mathbf{1 0}, \mathbf{3}) \oplus(\mathbf{1}, \mathbf{3})$ \\
\hline 53 & $\mathfrak{G} \mathfrak{u}(5) \oplus \mathfrak{G} \mathfrak{t}(2)$ & $(\overline{\mathbf{5}}, \mathbf{1}) \oplus(\mathbf{1 0}, \mathbf{1}) \oplus(\overline{\mathbf{5}}, \mathbf{2}) \oplus(\mathbf{1 0}, \mathbf{2}) \oplus(\mathbf{1}, \mathbf{2}) \oplus(\mathbf{1}, \mathbf{1})$ \\
\hline 54 & $\mathfrak{G H} \mathfrak{t}(5) \oplus \mathfrak{G} \mathfrak{t}(2)$ & $(\overline{5}, \mathbf{1}) \oplus(\mathbf{1 0}, \mathbf{1}) \oplus(\overline{\mathbf{5}}, \mathbf{2}) \oplus(\mathbf{1 0}, \mathbf{2}) \oplus(\mathbf{1}, \mathbf{3})$ \\
\hline 55 & $\mathfrak{S H} \mathfrak{t}(5) \oplus \mathfrak{S} \mathfrak{i t}(2)$ & $(\overline{\mathbf{5}}, \mathbf{1}) \oplus(\mathbf{1 0}, \mathbf{1})^{\oplus 3} \oplus(\overline{\mathbf{5}}, \mathbf{2}) \oplus(\mathbf{1}, \mathbf{1})^{\oplus 3}$ \\
\hline 56 & $\mathfrak{G} \mathfrak{u}(5) \oplus \mathfrak{G} \mathfrak{t}(2)$ & $(\mathbf{1 0}, \mathbf{1})^{\oplus 3} \oplus(\overline{\mathbf{5}}, \mathbf{3}) \oplus(\mathbf{1}, \mathbf{2}) \oplus(\mathbf{1 0}, \mathbf{1})$ \\
\hline 57 & $\mathfrak{G} \mathfrak{u}(5) \oplus \mathfrak{G} \mathfrak{i t}(2)$ & $(\mathbf{1 0}, \mathbf{1})^{\oplus 3} \oplus(\overline{\mathbf{5}}, \mathbf{3}) \oplus(\mathbf{1}, \mathbf{3})$ \\
\hline 58 & $\mathfrak{S} \mathfrak{u}(5) \oplus \mathfrak{G} \mathfrak{t}(2)$ & $(\overline{\mathbf{5}}, \mathbf{1})^{\oplus 3} \oplus(\mathbf{1 0}, \mathbf{1}) \oplus(\mathbf{1 0}, \mathbf{2}) \oplus(\mathbf{1}, \mathbf{2}) \oplus(\mathbf{1}, \mathbf{1})$ \\
\hline 59 & $\mathfrak{G} \mathfrak{i t}(5) \oplus \mathfrak{G} \mathfrak{t}(2)$ & $(\overline{\mathbf{5}}, \mathbf{1})^{\oplus 3} \oplus(\mathbf{1 0}, \mathbf{1}) \oplus(\mathbf{1 0}, \mathbf{2}) \oplus(\mathbf{1}, \mathbf{3})$ \\
\hline 60 & $\mathfrak{S} \mathfrak{i t}(5) \oplus \mathfrak{G} \mathfrak{i t}(2)$ & $(\overline{\mathbf{5}}, \mathbf{1}) \oplus(\mathbf{1 0}, \mathbf{1})^{\oplus 3} \oplus(\overline{\mathbf{5}}, \mathbf{2}) \oplus(\mathbf{1}, \mathbf{2}) \oplus(\mathbf{1}, \mathbf{1})$ \\
\hline 61 & $\mathfrak{S} \mathfrak{u}(5) \oplus \mathfrak{g} \mathfrak{t}(2)$ & $(\overline{\mathbf{5}}, \mathbf{1}) \oplus(\mathbf{1 0}, \mathbf{1})^{\oplus 3} \oplus(\overline{\mathbf{5}}, \mathbf{2}) \oplus(\mathbf{1}, \mathbf{3})$ \\
\hline 62 & $\mathfrak{G} \mathfrak{u}(5) \oplus \mathfrak{G} \mathfrak{i t}(2)$ & $(\overline{\mathbf{5}}, \mathbf{1})^{\oplus 3} \oplus(\mathbf{1 0}, \mathbf{1})^{\oplus 3} \oplus(\mathbf{1}, \mathbf{2}) \oplus(\mathbf{1}, \mathbf{1})$ \\
\hline 63 & $\mathfrak{S H} \mathfrak{t}(5) \oplus \mathfrak{S} \mathfrak{t}(2)$ & $(\overline{\mathbf{5}}, \mathbf{1})^{\oplus 3} \oplus(\mathbf{1 0}, \mathbf{1})^{\oplus 3} \oplus(\mathbf{1}, \mathbf{3})$ \\
\hline 64 & $\mathfrak{S} \mathfrak{t}(5)^{\oplus 2}$ & $(\overline{\mathbf{5}}, \mathbf{1})^{\oplus 2} \oplus(\mathbf{1 0}, \mathbf{1})^{\oplus 2} \oplus(\mathbf{1}, \overline{\mathbf{5}}) \oplus(\mathbf{1}, \mathbf{1 0}) \oplus(\mathbf{1}, \mathbf{1})^{\oplus 3}$ \\
\hline 65 & $\mathfrak{s} \mathfrak{t}(4) \oplus \mathfrak{s p}(6) \oplus \mathfrak{s} \mathfrak{i t}(2)$ & $(\overline{4}, \mathbf{6}, \mathbf{1}) \oplus(4,1,2)^{\oplus 3}$ \\
\hline 66 & $\mathfrak{s} \mathfrak{t}(4) \oplus \mathfrak{s p}(6) \oplus \mathfrak{s} \mathfrak{i t}(2)$ & $(\overline{\mathbf{4}}, \mathbf{6}, \mathbf{1}) \oplus(\mathbf{4}, \mathbf{1}, \mathbf{2})^{\oplus 3}$ \\
\hline 67 & $\mathfrak{s} \mathfrak{i t}(5) \oplus \mathfrak{S} \mathfrak{v}(10)^{\oplus 2}$ & $(\overline{5}, \mathbf{1}, \mathbf{1}) \oplus(\mathbf{1 0}, \mathbf{1}, \mathbf{1}) \oplus(\mathbf{1}, \mathbf{1 6}, \mathbf{1}) \oplus(\mathbf{1}, \mathbf{1}, \mathbf{1 6}) \oplus(\mathbf{1}, \mathbf{1}, \mathbf{1})$ \\
\hline 68 & $\mathfrak{S} \mathfrak{i t}(5) \oplus \mathfrak{g} \mathfrak{v}(10) \oplus \mathfrak{g} \mathfrak{i t}(2)$ & $(\mathbf{1}, \mathbf{1 6}, \mathbf{1}) \oplus(\overline{\mathbf{5}}, \mathbf{1}, \mathbf{2}) \oplus(\mathbf{1 0}, \mathbf{1}, \mathbf{2}) \oplus(\mathbf{1}, \mathbf{1 1}, \mathbf{1})^{\oplus 2}$ \\
\hline 69 & $\mathfrak{S} \mathfrak{t}(5) \oplus \mathfrak{g} \mathfrak{o}(10) \oplus \mathfrak{s} \mathfrak{u}(2)$ & $(\overline{5}, 1,1) \oplus(\mathbf{1 0}, 1,1) \oplus(1,16,2) \oplus(1,1,1)$ \\
\hline 70 & $\mathfrak{S} \mathfrak{t}(5) \oplus \mathfrak{g} \mathfrak{v}(10) \oplus \mathfrak{g} \mathfrak{i t}(2)$ & $(1,16,1) \oplus(\overline{5}, 1,2) \oplus(10,1,2) \oplus(1,1,2)$ \\
\hline 71 & $\mathfrak{S} \mathfrak{i t}(5) \oplus \mathfrak{g} \mathfrak{v}(10) \oplus \mathfrak{S} \mathfrak{i t}(2)$ & $(\overline{\mathbf{5}}, \mathbf{1}, \mathbf{1})^{\oplus 2} \oplus(\mathbf{1}, \mathbf{1 6}, \mathbf{1}) \oplus(\mathbf{1 0}, \mathbf{1}, \mathbf{2}) \oplus(\mathbf{1}, \mathbf{1}, \mathbf{1})^{\oplus 2}$ \\
\hline 72 & $\mathfrak{S} \mathfrak{t}(5) \oplus \mathfrak{g} \mathfrak{o}(10) \oplus \mathfrak{s} \mathfrak{i t}(2)$ & $(\mathbf{1 0}, \mathbf{1}, \mathbf{1})^{\oplus 2} \oplus(\mathbf{1}, \mathbf{1 6}, \mathbf{1}) \oplus(\overline{\mathbf{5}}, \mathbf{1}, \mathbf{2}) \oplus(\mathbf{1 0}, \mathbf{1}, \mathbf{1})^{\oplus 2}$ \\
\hline 73 & $\mathfrak{S} \mathfrak{u}(5) \oplus \mathfrak{g} \mathfrak{v}(10) \oplus \mathfrak{G} \mathfrak{u}(2)$ & $(\overline{\mathbf{5}}, \mathbf{1}, \mathbf{1})^{\oplus 2} \oplus(\mathbf{1}, \mathbf{1 6}, \mathbf{1}) \oplus(\mathbf{1 0}, \mathbf{1}, \mathbf{2}) \oplus(\mathbf{1}, \mathbf{1}, \mathbf{2})$ \\
\hline 74 & $\mathfrak{S} \mathfrak{t}(5) \oplus \mathfrak{g} \mathfrak{o}(10) \oplus \mathfrak{s} \mathfrak{i t}(2)$ & $(10,1,1)^{\oplus 2} \oplus(1,16,1) \oplus(\overline{5}, 1,2) \oplus(1,1,2)$ \\
\hline 75 & $\mathfrak{S} \mathfrak{t}(5) \oplus \mathfrak{g} \mathfrak{v}(10) \oplus \mathfrak{g} \mathfrak{i t}(2)$ & $(\overline{5}, \mathbf{1}, \mathbf{1})^{\oplus 2} \oplus(\mathbf{1 0}, \mathbf{1}, \mathbf{1})^{\oplus 2} \oplus(\mathbf{1}, \mathbf{1 6}, \mathbf{1}) \oplus(\mathbf{1}, \mathbf{1}, \mathbf{2})$ \\
\hline 76 & $\mathfrak{S} \mathfrak{i t}(5) \oplus \mathfrak{g} \mathfrak{i t}(2)^{\oplus 2}$ & $(\overline{\mathbf{5}}, \mathbf{3}, \mathbf{1}) \oplus(\mathbf{1 0}, \mathbf{1}, \mathbf{3}) \oplus(\mathbf{1}, \mathbf{1}, \mathbf{1})^{\oplus 3}$ \\
\hline 77 & $\mathfrak{S} \mathfrak{i t}(5) \oplus \mathfrak{g} \mathfrak{t}(2)^{\oplus 2}$ & $(\overline{\mathbf{5}}, \mathbf{1}, \mathbf{1}) \oplus(\overline{\mathbf{5}}, \mathbf{2}, \mathbf{1}) \oplus(\mathbf{1 0}, \mathbf{1}, \mathbf{3}) \oplus(\mathbf{1}, \mathbf{1}, \mathbf{1})^{\oplus 3}$ \\
\hline 78 & $\mathfrak{G} \mathfrak{t}(5) \oplus \mathfrak{g} \mathfrak{t}(2)^{\oplus 2}$ & $(\overline{\mathbf{5}}, \mathbf{3}, \mathbf{1}) \oplus(\mathbf{1 0}, \mathbf{1}, \mathbf{3}) \oplus(\mathbf{1}, \mathbf{1}, 2) \oplus(1,1,1)$ \\
\hline 79 & $\mathfrak{S} \mathfrak{i t}(5) \oplus \mathfrak{g} \mathfrak{t}(2)^{\oplus 2}$ & $(\overline{5}, 3,1) \oplus(\mathbf{1 0}, 1,3) \oplus(1,1,3)$ \\
\hline 80 & $\mathfrak{S} \mathfrak{t}(5) \oplus \mathfrak{G} \mathfrak{t}(2)^{\oplus 2}$ & $(\overline{5}, 3,1) \oplus(10,1,3) \oplus(1,2,1) \oplus(1,1,1)$ \\
\hline 81 & $\mathfrak{S} \mathfrak{i t}(5) \oplus \mathfrak{S} \mathfrak{t}(2)^{\oplus 2}$ & $(\overline{\mathbf{5}}, \mathbf{3}, \mathbf{1}) \oplus(\mathbf{1 0}, \mathbf{1}, \mathbf{3}) \oplus(\mathbf{1}, \mathbf{3}, \mathbf{1})$ \\
\hline 82 & $\mathfrak{S} \mathfrak{i t}(5) \oplus \mathfrak{g} \mathfrak{t}(2)^{\oplus 2}$ & $(\overline{\mathbf{5}}, \mathbf{3}, \mathbf{1}) \oplus(\mathbf{1 0}, \mathbf{3}, \mathbf{1}) \oplus(\mathbf{1}, 1,2) \oplus(\mathbf{1}, \mathbf{1}, \mathbf{1})$ \\
\hline 83 & $\mathfrak{S} \mathfrak{t}(5) \oplus \mathfrak{H} \mathfrak{t}(2)^{\oplus 2}$ & $(\overline{5}, 3,1) \oplus(\mathbf{1 0}, 3,1) \oplus(1,1,3)$ \\
\hline 84 & $\mathfrak{S} \mathfrak{t}(5) \oplus \mathfrak{g} \mathfrak{t}(2)^{\oplus 2}$ & $(\mathbf{1 0}, \mathbf{1}, \mathbf{1}) \oplus(\overline{\mathbf{5}}, \mathbf{3}, \mathbf{1}) \oplus(\mathbf{1 0}, \mathbf{1}, \mathbf{2}) \oplus(\mathbf{1 0}, \mathbf{1}, \mathbf{1})^{\oplus 3}$ \\
\hline 85 & $\mathfrak{S} \mathfrak{i t}(5) \oplus \mathfrak{s} \mathfrak{u}(2)^{\oplus 2}$ & $(\overline{\mathbf{5}}, \mathbf{1}, \mathbf{1}) \oplus(\overline{\mathbf{5}}, \mathbf{2}, \mathbf{1}) \oplus(\mathbf{1 0}, \mathbf{1}, \mathbf{3}) \oplus(\mathbf{1}, \mathbf{1}, \mathbf{2}) \oplus(\mathbf{1}, \mathbf{1}, \mathbf{1})$ \\
\hline
\end{tabular}


TABLE II. (Continued)

Nonmaximal and Nonminimal algebras

\begin{tabular}{|c|c|c|}
\hline & Algebra & Fermion representations corresponding to $\beta$ \\
\hline 86 & $\mathfrak{G} \mathfrak{i t}(5) \oplus \mathfrak{G} \mathfrak{t}(2)^{\oplus 2}$ & $(\overline{\mathbf{5}}, \mathbf{1}, \mathbf{1}) \oplus(\overline{\mathbf{5}}, \mathbf{2}, \mathbf{1}) \oplus(\mathbf{1 0}, \mathbf{1}, \mathbf{3}) \oplus(\mathbf{1}, \mathbf{1}, \mathbf{3})$ \\
\hline 87 & $\mathfrak{G} \mathfrak{i t}(5) \oplus \mathfrak{G} \mathfrak{t}(2)^{\oplus 2}$ & $(\overline{\mathbf{5}}, \mathbf{1}, \mathbf{1}) \oplus(\overline{\mathbf{5}}, \mathbf{2}, \mathbf{1}) \oplus(\mathbf{1 0}, \mathbf{1}, \mathbf{3}) \oplus(\mathbf{1}, \mathbf{2}, \mathbf{1}) \oplus(\mathbf{1}, \mathbf{1}, \mathbf{1})$ \\
\hline 88 & $\mathfrak{G} \mathfrak{i t}(5) \oplus \mathfrak{s} \mathfrak{i t}(2)^{\oplus 2}$ & $(\overline{\mathbf{5}}, \mathbf{1}, \mathbf{1}) \oplus(\overline{\mathbf{5}}, \mathbf{2}, \mathbf{1}) \oplus(\mathbf{1 0}, \mathbf{1}, \mathbf{3}) \oplus(\mathbf{1}, \mathbf{3}, \mathbf{1})$ \\
\hline 89 & $\mathfrak{s} \mathfrak{u}(5) \oplus \mathfrak{s} \mathfrak{t}(2) \oplus 2$ & $(\overline{\mathbf{5}}, \mathbf{1}, \mathbf{1}) \oplus(\overline{\mathbf{5}}, \mathbf{2}, \mathbf{1}) \oplus(\mathbf{1 0}, \mathbf{3}, \mathbf{1}) \oplus(\mathbf{1}, \mathbf{1}, \mathbf{2}) \oplus(\mathbf{1}, \mathbf{1}, \mathbf{1})$ \\
\hline 90 & $\mathfrak{S} \mathfrak{u}(5) \oplus \mathfrak{s} \mathfrak{t}(2)^{\oplus 2}$ & $(\overline{\mathbf{5}}, \mathbf{1}, \mathbf{1}) \oplus(\overline{\mathbf{5}}, \mathbf{2}, \mathbf{1}) \oplus(\mathbf{1 0}, \mathbf{3}, \mathbf{1}) \oplus(\mathbf{1}, \mathbf{1}, \mathbf{3})$ \\
\hline 91 & $\mathfrak{G} \mathfrak{i t}(5) \oplus \mathfrak{s} \mathfrak{t}(2)^{\oplus 2}$ & $(\overline{\mathbf{5}}, \mathbf{1}, \mathbf{1}) \oplus(\mathbf{1 0}, \mathbf{1}, \mathbf{1}) \oplus(\overline{\mathbf{5}}, \mathbf{2}, \mathbf{1}) \oplus(\mathbf{1 0}, \mathbf{1}, \mathbf{2}) \oplus(\mathbf{1}, \mathbf{1}, \mathbf{1})^{\oplus 3}$ \\
\hline 92 & $\mathfrak{G} \mathfrak{u}(5) \oplus \mathfrak{G} \mathfrak{t}(2)^{\oplus 2}$ & $(10,1,1) \oplus(\overline{5}, 3,1) \oplus(10,1,2) \oplus(1,1,2) \oplus(10,1,1)$ \\
\hline 93 & $\mathfrak{G} \mathfrak{H}(5) \oplus \mathfrak{G} \mathfrak{t}(2)^{\oplus 2}$ & $(\mathbf{1 0}, \mathbf{1}, \mathbf{1}) \oplus(\overline{\mathbf{5}}, \mathbf{3}, \mathbf{1}) \oplus(\mathbf{1 0}, \mathbf{1}, \mathbf{2}) \oplus(\mathbf{1}, \mathbf{1}, \mathbf{3})$ \\
\hline 94 & $\mathfrak{G} \mathfrak{i t}(5) \oplus \mathfrak{s} \mathfrak{t}(2)^{\oplus 2}$ & $(\mathbf{1 0}, \mathbf{1}, \mathbf{1}) \oplus(\overline{\mathbf{5}}, \mathbf{3}, \mathbf{1}) \oplus(\mathbf{1 0}, \mathbf{1}, \mathbf{2}) \oplus(\mathbf{1}, \mathbf{2}, \mathbf{1}) \oplus(\mathbf{1 0}, \mathbf{1}, \mathbf{1})$ \\
\hline 95 & $\mathfrak{S} \mathfrak{u}(5) \oplus \mathfrak{s} \mathfrak{t}(2)^{\oplus 2}$ & $(\mathbf{1 0}, \mathbf{1}, \mathbf{1}) \oplus(\overline{\mathbf{5}}, \mathbf{3}, \mathbf{1}) \oplus(\mathbf{1 0}, \mathbf{1}, \mathbf{2}) \oplus(\mathbf{1}, \mathbf{3}, \mathbf{1})$ \\
\hline 96 & $\mathfrak{G} \mathfrak{u}(5) \oplus \mathfrak{G} \mathfrak{t}(2)^{\oplus 2}$ & $(10,1,1) \oplus(\overline{5}, 3,1) \oplus(10,2,1) \oplus(1,1,2) \oplus(10,1,1)$ \\
\hline 97 & $\mathfrak{G} \mathfrak{i t}(5) \oplus \mathfrak{G} \mathfrak{t}(2)^{\oplus 2}$ & $(\mathbf{1 0}, \mathbf{1}, \mathbf{1}) \oplus(\overline{\mathbf{5}}, \mathbf{3}, \mathbf{1}) \oplus(\mathbf{1 0}, \mathbf{2}, \mathbf{1}) \oplus(\mathbf{1}, \mathbf{1}, \mathbf{3})$ \\
\hline 98 & $\mathfrak{G} \mathfrak{u}(5) \oplus \mathfrak{s} \mathfrak{t}(2)^{\oplus 2}$ & $(\overline{\mathbf{5}}, \mathbf{1}, \mathbf{1})^{\oplus 3} \oplus(\mathbf{1 0}, \mathbf{3}, \mathbf{1}) \oplus(\mathbf{1}, \mathbf{1}, \mathbf{2}) \oplus(\mathbf{1}, \mathbf{1}, \mathbf{1})$ \\
\hline 99 & $\mathfrak{G} \mathfrak{u}(5) \oplus \mathfrak{s} \mathfrak{i t}(2)^{\oplus 2}$ & $(\overline{\mathbf{5}}, \mathbf{1}, \mathbf{1})^{\oplus 3} \oplus(\mathbf{1 0}, \mathbf{3}, \mathbf{1}) \oplus(\mathbf{1}, \mathbf{1}, \mathbf{3})$ \\
\hline 100 & $\mathfrak{S} \mathfrak{i t}(5) \oplus \mathfrak{s} \mathfrak{i t}(2)^{\oplus 2}$ & $(\overline{5}, 1,1) \oplus(\mathbf{1 0}, 1,1) \oplus(\overline{5}, 2,1) \oplus(\mathbf{1 0}, 1,2) \oplus(1,1,2) \oplus(1,1,1)$ \\
\hline 101 & $\mathfrak{G} \mathfrak{u}(5) \oplus \mathfrak{G} \mathfrak{t}(2)^{\oplus 2}$ & $(\overline{\mathbf{5}}, \mathbf{1}, \mathbf{1}) \oplus(\mathbf{1 0}, \mathbf{1}, \mathbf{1}) \oplus(\overline{\mathbf{5}}, \mathbf{2}, \mathbf{1}) \oplus(\mathbf{1 0}, \mathbf{1}, \mathbf{2}) \oplus(\mathbf{1}, \mathbf{1}, \mathbf{3})$ \\
\hline 102 & $\mathfrak{G} \mathfrak{i t}(5) \oplus \mathfrak{s} \mathfrak{t}(2)^{\oplus 2}$ & $(\overline{\mathbf{5}}, \mathbf{1}, \mathbf{1}) \oplus(\mathbf{1 0}, \mathbf{1}, \mathbf{1}) \oplus(\overline{\mathbf{5}}, \mathbf{2}, \mathbf{1}) \oplus(\mathbf{1 0}, \mathbf{1}, \mathbf{2}) \oplus(\mathbf{1}, \mathbf{2}, \mathbf{1}) \oplus(\mathbf{1}, \mathbf{1}, \mathbf{1})$ \\
\hline 103 & $\mathfrak{G} \mathfrak{u}(5) \oplus \mathfrak{s} \mathfrak{t}(2)^{\oplus 2}$ & $(\overline{\mathbf{5}}, \mathbf{1}, \mathbf{1}) \oplus(\mathbf{1 0}, \mathbf{1}, \mathbf{1}) \oplus(\overline{\mathbf{5}}, \mathbf{2}, \mathbf{1}) \oplus(\mathbf{1 0}, \mathbf{1}, \mathbf{2}) \oplus(\mathbf{1}, \mathbf{3}, \mathbf{1})$ \\
\hline 104 & $\mathfrak{G} \mathfrak{i t}(5) \oplus \mathfrak{s} \mathfrak{i t}(2)^{\oplus 2}$ & $(\overline{5}, 1,1) \oplus(\mathbf{1 0}, 1,1) \oplus(\overline{5}, 2,1) \oplus(\mathbf{1 0}, 2,1) \oplus(1,1,2) \oplus(1,1,1)$ \\
\hline 105 & $\mathfrak{G} \mathfrak{i t}(5) \oplus \mathfrak{s} \mathfrak{i t}(2)^{\oplus 2}$ & $(\overline{5}, \mathbf{1}, \mathbf{1}) \oplus(\mathbf{1 0}, \mathbf{1}, \mathbf{1}) \oplus(\overline{\mathbf{5}}, \mathbf{2}, \mathbf{1}) \oplus(\mathbf{1 0}, \mathbf{2}, \mathbf{1}) \oplus(\mathbf{1}, \mathbf{1}, \mathbf{3})$ \\
\hline 106 & $\mathfrak{G} \mathfrak{i t}(5) \oplus \mathfrak{s} \mathfrak{t}(2)^{\oplus 2}$ & $(\mathbf{1 0}, \mathbf{1}, \mathbf{1})^{\oplus 3} \oplus(\overline{\mathbf{5}}, \mathbf{3}, \mathbf{1}) \oplus(\mathbf{1}, \mathbf{1}, \mathbf{2}) \oplus(\mathbf{1 0}, \mathbf{1}, \mathbf{1})$ \\
\hline 107 & $\mathfrak{G} \mathfrak{i t}(5) \oplus \mathfrak{s} \mathfrak{t}(2)^{\oplus 2}$ & $(\mathbf{1 0}, \mathbf{1}, \mathbf{1})^{\oplus 3} \oplus(\overline{\mathbf{5}}, \mathbf{3}, \mathbf{1}) \oplus(\mathbf{1}, \mathbf{1}, \mathbf{3})$ \\
\hline 108 & $\mathfrak{S} \mathfrak{u}(5) \oplus \mathfrak{s} \mathfrak{i t}(2)^{\oplus 2}$ & $(\overline{\mathbf{5}}, \mathbf{1}, \mathbf{1})^{\oplus 3} \oplus(\mathbf{1 0}, \mathbf{1}, \mathbf{1}) \oplus(\mathbf{1 0}, \mathbf{2}, \mathbf{1}) \oplus(\mathbf{1}, \mathbf{1}, \mathbf{2}) \oplus(\mathbf{1}, \mathbf{1}, \mathbf{1})$ \\
\hline 109 & $\mathfrak{G} \mathfrak{i t}(5) \oplus \mathfrak{s} \mathfrak{t}(2)^{\oplus 2}$ & $(\overline{\mathbf{5}}, \mathbf{1}, \mathbf{1}) \oplus^{\top} \oplus(\mathbf{1 0}, \mathbf{1}, \mathbf{1}) \oplus(\mathbf{1 0}, \mathbf{2}, \mathbf{1}) \oplus(\mathbf{1}, \mathbf{1}, \mathbf{3})$ \\
\hline 110 & $\mathfrak{S} \mathfrak{i t}(5) \oplus \mathfrak{s} \mathfrak{t}(2)^{\oplus 2}$ & $(\overline{\mathbf{5}}, \mathbf{1}, \mathbf{1}) \oplus(\mathbf{1 0}, \mathbf{1}, \mathbf{1})^{\oplus 3} \oplus(\overline{\mathbf{5}}, \mathbf{2}, \mathbf{1}) \oplus(\mathbf{1}, \mathbf{1}, \mathbf{2}) \oplus(\mathbf{1}, \mathbf{1}, \mathbf{1})$ \\
\hline 111 & $\mathfrak{G} \mathfrak{u}(5) \oplus \mathfrak{s} \mathfrak{t}(2)^{\oplus 2}$ & $(\overline{\mathbf{5}}, \mathbf{1}, \mathbf{1}) \oplus(\mathbf{1 0}, \mathbf{1}, \mathbf{1})^{\oplus 3} \oplus(\overline{\mathbf{5}}, \mathbf{2}, \mathbf{1}) \oplus(\mathbf{1}, \mathbf{1}, \mathbf{3})$ \\
\hline 112 & $\mathfrak{G} \mathfrak{u}(5)^{\oplus 2} \oplus \mathfrak{S} \mathfrak{o}(10)$ & $(\overline{\mathbf{5}}, \mathbf{1}, \mathbf{1}) \oplus(\mathbf{1 0}, \mathbf{1}, \mathbf{1}) \oplus(\mathbf{1}, \overline{\mathbf{5}}, \mathbf{1}) \oplus(\mathbf{1}, \mathbf{1 0}, \mathbf{1}) \oplus(\mathbf{1}, \mathbf{1}, \mathbf{1 6}) \oplus(\mathbf{1}, \mathbf{1}, \mathbf{1})^{\oplus 2}$ \\
\hline 113 & $\mathfrak{S} \mathfrak{u}(5)^{\oplus 2} \oplus \mathfrak{g} \mathfrak{t}(2)$ & $(\mathbf{1}, \overline{\mathbf{5}}, \mathbf{1}) \oplus(\mathbf{1}, \mathbf{1 0}, \mathbf{1}) \oplus(\overline{\mathbf{5}}, \mathbf{1}, \mathbf{2}) \oplus(\mathbf{1 0}, \mathbf{1}, \mathbf{2}) \oplus(\mathbf{1}, \mathbf{1}, \mathbf{1})^{\oplus 3}$ \\
\hline 114 & $\mathfrak{G} \mathfrak{u}(5)^{\oplus 2} \oplus \mathfrak{G} \mathfrak{i t}(2)$ & $(\mathbf{1}, \overline{\mathbf{5}}, \mathbf{1}) \oplus(\mathbf{1}, \mathbf{1 0}, \mathbf{1}) \oplus(\overline{\mathbf{5}}, \mathbf{1}, \mathbf{2}) \oplus(\mathbf{1 0}, \mathbf{1}, \mathbf{2}) \oplus(\mathbf{1}, \mathbf{1}, \mathbf{2}) \oplus(\mathbf{1}, \mathbf{1}, \mathbf{1})$ \\
\hline 115 & $\mathfrak{G} \mathfrak{i t}(5)^{\oplus 2} \oplus \mathfrak{g} \mathfrak{i t}(2)$ & $(\mathbf{1}, \overline{\mathbf{5}}, \mathbf{1}) \oplus(\mathbf{1}, \mathbf{1 0}, \mathbf{1}) \oplus(\overline{\mathbf{5}}, \mathbf{1}, \mathbf{2}) \oplus(\mathbf{1 0}, \mathbf{1}, \mathbf{2}) \oplus(\mathbf{1}, \mathbf{1}, \mathbf{3})$ \\
\hline 116 & $\mathfrak{G} \mathfrak{u}(5)^{\oplus 2} \oplus \mathfrak{s} \mathfrak{i t}(2)$ & $(\overline{\mathbf{5}}, \mathbf{1}, \mathbf{1})^{\oplus 2} \oplus(\mathbf{1}, \overline{\mathbf{5}}, \mathbf{1}) \oplus(\mathbf{1}, \mathbf{1 0}, \mathbf{1}) \oplus(\mathbf{1 0}, \mathbf{1}, \mathbf{2}) \oplus(\mathbf{1}, \mathbf{1}, \mathbf{1})^{\oplus 3}$ \\
\hline 117 & $\mathfrak{S} \mathfrak{u}(5)^{\oplus 2} \oplus \mathfrak{s} \mathfrak{i t}(2)$ & $(\mathbf{1 0}, \mathbf{1}, \mathbf{1})^{\oplus 2} \oplus(\mathbf{1}, \overline{\mathbf{5}}, \mathbf{1}) \oplus(\mathbf{1}, \mathbf{1 0}, \mathbf{1}) \oplus(\overline{\mathbf{5}}, \mathbf{1}, \mathbf{2}) \oplus(\mathbf{1 0}, \mathbf{1}, \mathbf{1})^{\oplus 3}$ \\
\hline 118 & $\mathfrak{G} \mathfrak{i t}(5)^{\oplus 2} \oplus \mathfrak{g} \mathfrak{i t}(2)$ & $(\overline{\mathbf{5}}, \mathbf{1}, \mathbf{1})^{\oplus 2} \oplus(\mathbf{1}, \overline{\mathbf{5}}, \mathbf{1}) \oplus(\mathbf{1}, \mathbf{1 0}, \mathbf{1}) \oplus(\mathbf{1 0}, \mathbf{1}, \mathbf{2}) \oplus(\mathbf{1}, \mathbf{1}, \mathbf{2}) \oplus(\mathbf{1}, \mathbf{1}, \mathbf{1})$ \\
\hline 119 & $\mathfrak{G} \mathfrak{i t}(5)^{\oplus 2} \oplus \mathfrak{G} \mathfrak{i t}(2)$ & $(\overline{\mathbf{5}}, \mathbf{1}, \mathbf{1})^{\oplus 2} \oplus(\mathbf{1}, \overline{\mathbf{5}}, \mathbf{1}) \oplus(\mathbf{1}, \mathbf{1 0}, \mathbf{1}) \oplus(\mathbf{1 0}, \mathbf{1}, \mathbf{2}) \oplus(\mathbf{1}, \mathbf{1}, \mathbf{3})$ \\
\hline 120 & $\mathfrak{G} \mathfrak{i t}(5)^{\oplus 2} \oplus \mathfrak{g} \mathfrak{i t}(2)$ & $(\mathbf{1 0}, \mathbf{1}, \mathbf{1})^{\oplus 2} \oplus(\mathbf{1}, \overline{\mathbf{5}}, \mathbf{1}) \oplus(\mathbf{1}, \mathbf{1 0}, \mathbf{1}) \oplus(\overline{\mathbf{5}}, \mathbf{1}, \mathbf{2}) \oplus(\mathbf{1}, \mathbf{1}, \mathbf{2}) \oplus(\mathbf{1 0}, \mathbf{1}, \mathbf{1})$ \\
\hline 121 & $\mathfrak{G} \mathfrak{i t}(5)^{\oplus 2} \oplus \mathfrak{g} \mathfrak{i t}(2)$ & $(\mathbf{1 0}, \mathbf{1}, \mathbf{1})^{\oplus 2} \oplus(\mathbf{1}, \overline{\mathbf{5}}, \mathbf{1}) \oplus(\mathbf{1}, \mathbf{1 0}, \mathbf{1}) \oplus(\overline{\mathbf{5}}, \mathbf{1}, \mathbf{2}) \oplus(\mathbf{1}, \mathbf{1}, \mathbf{3})$ \\
\hline 122 & $\mathfrak{S} \mathfrak{u}(5)^{\oplus 2} \oplus \mathfrak{s} \mathfrak{i t}(2)$ & $(\overline{\mathbf{5}}, \mathbf{1}, \mathbf{1})^{\oplus 2} \oplus(\mathbf{1 0}, \mathbf{1}, \mathbf{1})^{\oplus 2} \oplus(\mathbf{1}, \overline{\mathbf{5}}, \mathbf{1}) \oplus(\mathbf{1}, \mathbf{1 0}, \mathbf{1}) \oplus(\mathbf{1}, \mathbf{1}, \mathbf{2}) \oplus(\mathbf{1}, \mathbf{1}, \mathbf{1})$ \\
\hline 123 & $\mathfrak{G} \mathfrak{i t}(5)^{\oplus 2} \oplus \mathfrak{G} \mathfrak{i t}(2)$ & $(\overline{\mathbf{5}}, \mathbf{1}, \mathbf{1})^{\oplus 2} \oplus(\mathbf{1 0}, \mathbf{1}, \mathbf{1})^{\oplus 2} \oplus(\mathbf{1}, \overline{\mathbf{5}}, \mathbf{1}) \oplus(\mathbf{1}, \mathbf{1 0}, \mathbf{1}) \oplus(\mathbf{1}, \mathbf{1}, \mathbf{3})$ \\
\hline 124 & $\mathfrak{G} \mathfrak{i t}(5)^{\oplus 3}$ & $(\overline{\mathbf{5}}, \mathbf{1}, \mathbf{1}) \oplus(\mathbf{1 0}, \mathbf{1}, \mathbf{1}) \oplus(\mathbf{1}, \overline{\mathbf{5}}, \mathbf{1}) \oplus(\mathbf{1}, \mathbf{1 0}, \mathbf{1}) \oplus(\mathbf{1}, \mathbf{1}, \overline{\mathbf{5}}) \oplus(\mathbf{1}, \mathbf{1}, \mathbf{1 0}) \oplus(\mathbf{1}, \mathbf{1}, \mathbf{1})^{\oplus 3}$ \\
\hline 125 & $\mathfrak{G} \mathfrak{i t}(3) \oplus \mathfrak{g} \mathfrak{t}(4) \oplus \mathfrak{g} \mathfrak{t}(2)^{\oplus 2}$ & $(\overline{3}, \overline{4}, 2,1) \oplus(3,4,1,2)$ \\
\hline 126 & $\mathfrak{G} \mathfrak{u}(4) \oplus \mathfrak{S} \mathfrak{o}(10) \oplus \mathfrak{S} \mathfrak{i t}(2)^{\oplus 2}$ & $(\mathbf{1}, \mathbf{1 6}, \mathbf{1}, \mathbf{1})^{\oplus 2} \oplus(\overline{\mathbf{4}}, \mathbf{1}, \mathbf{2}, \mathbf{1}) \oplus(\mathbf{4}, \mathbf{1}, \mathbf{1}, \mathbf{2})$ \\
\hline 127 & $\mathfrak{S} \mathfrak{i t}(4) \oplus \mathfrak{S} \mathfrak{o}(10) \oplus \mathfrak{S} \mathfrak{t}(2)^{\oplus 2}$ & $(1,16,1,1) \oplus(\overline{4}, 1,2,1)^{\oplus 2} \oplus(4,1,1,2)^{\oplus 2}$ \\
\hline 128 & $\mathfrak{G} \mathfrak{t}(4) \oplus \mathfrak{g p}(4) \oplus \mathfrak{S} \mathfrak{v}(10) \oplus \mathfrak{G} \mathfrak{t}(2)$ & $(\underline{4}, 4,1,1) \oplus(1,1,16,1) \oplus(4,1,1,2)^{\oplus 2}$ \\
\hline 129 & $\mathfrak{S} \mathfrak{i t}(4) \oplus \mathfrak{g} \mathfrak{p}(4) \oplus \mathfrak{S} \mathfrak{v}(10) \oplus \mathfrak{s} \mathfrak{t}(2)$ & $(\overline{4}, 4,1,1) \oplus(1,1,16,1) \oplus(4,1,1,2) \oplus 2$ \\
\hline 130 & $\mathfrak{S} \mathfrak{i t}(4) \oplus \mathfrak{g} \mathfrak{p}(4) \oplus \mathfrak{s p}(6) \oplus \mathfrak{g} \mathfrak{t}(2)$ & $(\overline{4}, 4,1,1) \oplus(4,1,6,1) \oplus(\overline{4}, 1,1,2)$ \\
\hline 131 & $\mathfrak{s} \mathfrak{u}(4) \oplus \mathfrak{s p}(4) \oplus \mathfrak{s p}(6) \oplus \mathfrak{g} \mathfrak{u}(2)$ & $(\overline{4}, 4,1,1) \oplus(4,1,6,1) \oplus(\overline{4}, 1,1,2)$ \\
\hline 132 & $\mathfrak{G} \mathfrak{i t}(4) \oplus \mathfrak{s} \mathfrak{p}(4) \oplus \mathfrak{s} \mathfrak{i t}(2)^{\oplus 2}$ & $(\underline{\mathbf{4}}, \mathbf{4}, \mathbf{1}, \mathbf{1}) \oplus(\underline{\mathbf{4}}, \mathbf{1}, \mathbf{2}, \mathbf{1}) \oplus(\mathbf{4}, \mathbf{1}, \mathbf{1}, \mathbf{2})^{\oplus 3}$ \\
\hline 133 & $\mathfrak{G} \mathfrak{t}(4) \oplus \mathfrak{g} \mathfrak{p}(4) \oplus \mathfrak{s} \mathfrak{t}(2)^{\oplus 2}$ & $(\overline{\mathbf{4}}, \mathbf{4}, \mathbf{1}, \mathbf{1}) \oplus(\overline{\mathbf{4}}, \mathbf{1}, \mathbf{2}, \mathbf{1}) \oplus(\mathbf{4}, \mathbf{1}, \mathbf{1}, \mathbf{2})^{\oplus 3}$ \\
\hline 134 & $\mathfrak{G} \mathfrak{t}(4) \oplus \mathfrak{S p}(6) \oplus \mathfrak{g} \mathfrak{t}(2)^{\oplus 2}$ & $(\underline{\mathbf{4}}, \mathbf{6}, 1,1) \oplus(\mathbf{4}, 1,3,2)$ \\
\hline 135 & $\mathfrak{S} \mathfrak{t}(4) \oplus \mathfrak{s} \mathfrak{p}(6) \oplus \mathfrak{g} \mathfrak{t}(2)^{\oplus 2}$ & $(\overline{\mathbf{4}}, \mathbf{6}, \mathbf{1}, \mathbf{1}) \oplus(\mathbf{4}, \mathbf{1}, \mathbf{3}, \mathbf{2})$ \\
\hline 136 & $\mathfrak{G} \mathfrak{t}(4) \oplus \mathfrak{g} \mathfrak{p}(6) \oplus \mathfrak{G} \mathfrak{t}(2)^{\oplus 2}$ & $(\overline{4}, 6,1,1) \oplus(4,1,2,2) \oplus(4,1,2,1)$ \\
\hline 137 & $\mathfrak{G} \mathfrak{t}(4) \oplus \mathfrak{s} \mathfrak{p}(6) \oplus \mathfrak{s} \mathfrak{t}(2)^{\oplus 2}$ & $(\overline{4}, 6,1,1) \oplus(4,1,2,2) \oplus(4,1,2,1)$ \\
\hline 138 & $\mathfrak{G} \mathfrak{t}(4) \oplus \mathfrak{g} \mathfrak{p}(6) \oplus \mathfrak{g} \mathfrak{t}(2)^{\oplus 2}$ & $(\underline{4}, 6,1,1) \oplus(4,1,2,1)^{\oplus 2} \oplus(4,1,1,2)$ \\
\hline 139 & $\mathfrak{G} \mathfrak{t}(4) \oplus \mathfrak{S} \mathfrak{p}(6) \oplus \mathfrak{G} \mathfrak{t}(2)^{\oplus 2}$ & $(\overline{4}, 6,1,1) \oplus(4,1,2,1)^{\oplus 2} \oplus(4,1,1,2)$ \\
\hline
\end{tabular}


TABLE II. (Continued)

Nonmaximal and Nonminimal algebras

\begin{tabular}{|c|c|}
\hline Algebra & Fermion representations corresponding to $\beta$ \\
\hline $140 \mathfrak{g} \mathfrak{t}(4) \oplus \mathfrak{g} \mathfrak{i t}(2)^{\oplus 3}$ & $(4,3,2,1) \oplus(\overline{4}, 3,1,2)$ \\
\hline $141 \mathfrak{g} \mathfrak{t}(4) \oplus \mathfrak{g} \mathfrak{t}(2)^{\oplus 3}$ & $(4,2,2,1) \oplus(\overline{4}, 3,1,2) \oplus(4,1,2,1)$ \\
\hline $142 \mathfrak{g} \mathfrak{t}(4) \oplus \mathfrak{g} \mathfrak{t}(2)^{\oplus 3}$ & $(4,2,2,1) \oplus(\overline{4}, 3,1,2) \oplus(4,1,2,1)$ \\
\hline $143 \mathfrak{g} \mathfrak{t}(4) \oplus \mathfrak{g} \mathfrak{u}(2)^{\oplus 3}$ & $(4,2,2,1) \oplus(\overline{4}, 2,1,2) \oplus(4,1,2,1) \oplus(\overline{4}, 1,1,2)$ \\
\hline $144 \mathfrak{g} \mathfrak{t}(4) \oplus \mathfrak{g} \mathfrak{u}(2)^{\oplus 3}$ & $(4,3,2,1) \oplus(\overline{4}, 1,1,2) \oplus 3$ \\
\hline $145 \mathfrak{g} \mathfrak{t}(4) \oplus \mathfrak{g} \mathfrak{t}(2)^{\oplus 3}$ & $(\mathbf{4}, \mathbf{3}, \mathbf{2}, \mathbf{1}) \oplus(\overline{4}, \mathbf{1}, \mathbf{1}, \mathbf{2})^{\oplus 3}$ \\
\hline $146 \mathfrak{g} \mathfrak{u}(4) \oplus \mathfrak{g} \mathfrak{u}(2)^{\oplus 3}$ & $(4,2,2,1) \oplus(4,2,1,1) \oplus(\overline{4}, 1,1,2)^{\oplus 3}$ \\
\hline $147 \mathfrak{g} \mathfrak{t}(4) \oplus \mathfrak{g} \mathfrak{u}(2)^{\oplus 3}$ & $(4,2,2,1) \oplus(4,2,1,1) \oplus(\overline{4}, 1,1,2)^{\oplus 3}$ \\
\hline $148 \mathfrak{g} \mathfrak{t}(4) \oplus \mathfrak{g} \mathfrak{u}(2)^{\oplus 3}$ & $(\overline{4}, 2,1,1)^{\oplus 3} \oplus(4,1,2,1)^{\oplus 2} \oplus(4,1,1,2)$ \\
\hline $149 \mathfrak{g} \mathfrak{t}(4) \oplus \mathfrak{g} \mathfrak{i t}(2)^{\oplus 3}$ & $(\overline{\mathbf{4}}, \mathbf{2}, \mathbf{1}, \mathbf{1})^{\oplus 3} \oplus(\mathbf{4}, \mathbf{1}, \mathbf{2}, \mathbf{1})^{\oplus 2} \oplus(\mathbf{4}, \mathbf{1}, \mathbf{1}, 2)$ \\
\hline $150 \mathfrak{S} \mathfrak{t}(4)^{\oplus 2} \oplus \mathfrak{s} \mathfrak{p}(4) \oplus \mathfrak{G} \mathfrak{t}(2)$ & $(\overline{4}, 6,1,1) \oplus(4,1,4,1) \oplus(4,1,1,2)$ \\
\hline $151 \mathfrak{g} \mathfrak{i t}(4)^{\oplus 2} \oplus \mathfrak{g} \mathfrak{u}(2)^{\oplus 2}$ & $(\overline{4}, 6,1,1) \oplus(4,1,3,2)$ \\
\hline $152 \mathfrak{g} \mathfrak{u}(4)^{\oplus 2} \oplus \mathfrak{G} \mathfrak{t}(2)^{\oplus 2}$ & $(\overline{4}, 6,1,1) \oplus(4,1,2,2) \oplus(4,1,2,1)$ \\
\hline $153 \mathfrak{g} \mathfrak{u}(4)^{\oplus 2} \oplus \mathfrak{G} \mathfrak{t}(2)^{\oplus 2}$ & $(\overline{4}, 6,1,1) \oplus(4,1,2,1)^{\oplus 2} \oplus(4,1,1,2)$ \\
\hline $154 \mathfrak{S} \mathfrak{i t}(4)^{\oplus 2} \oplus \mathfrak{G} \mathfrak{t}(2)^{\oplus 2}$ & $(\overline{\mathbf{4}}, \mathbf{1}, \mathbf{2}, \mathbf{1})^{\oplus 2} \oplus(\mathbf{1}, \overline{\mathbf{4}}, \mathbf{2}, \mathbf{1}) \oplus(\mathbf{4}, \mathbf{1}, \mathbf{1}, \mathbf{2})^{\oplus 2} \oplus(\mathbf{1}, \mathbf{4}, \mathbf{1}, \mathbf{2})$ \\
\hline $155 \mathfrak{G} \mathfrak{u}(4) \oplus \mathfrak{S} \mathfrak{t}(5) \oplus \mathfrak{S} \mathfrak{t}(4)^{\oplus 2}$ & $(\overline{\mathbf{4}}, \mathbf{1}, \mathbf{4}, \mathbf{1}) \oplus(\mathbf{4}, \mathbf{1}, \mathbf{1}, \mathbf{4}) \oplus(\mathbf{1}, \overline{\mathbf{5}}, \mathbf{1}, \mathbf{1}) \oplus(\mathbf{1}, \mathbf{1 0}, \mathbf{1}, \mathbf{1}) \oplus(\mathbf{1}, \mathbf{1}, \mathbf{1}, \mathbf{1})$ \\
\hline $156 \mathfrak{g} \mathfrak{t}(4) \oplus \mathfrak{G} \mathfrak{i t}(5) \oplus \mathfrak{S p}(4) \oplus \mathfrak{G} \mathfrak{t}(2)$ & $(\overline{\mathbf{4}}, \mathbf{1}, \mathbf{4}, \mathbf{1}) \oplus(\mathbf{1}, \overline{\mathbf{5}}, \mathbf{1}, \mathbf{1}) \oplus(\mathbf{1}, \mathbf{1 0}, \mathbf{1}, \mathbf{1}) \oplus(\mathbf{4}, \mathbf{1}, \mathbf{1}, \mathbf{2}) \oplus^{\oplus 2} \oplus(\mathbf{1}, \mathbf{1}, \mathbf{1}, \mathbf{1})$ \\
\hline $157 \mathfrak{g} \mathfrak{i t}(4) \oplus \mathfrak{g} \mathfrak{i t}(5) \oplus \mathfrak{s} \mathfrak{p}(4) \oplus \mathfrak{s} \mathfrak{i t}(2)$ & $(\overline{\mathbf{4}}, \mathbf{1}, \mathbf{4}, \mathbf{1}) \oplus(\mathbf{1}, \overline{\mathbf{5}}, \mathbf{1}, \mathbf{1}) \oplus(\mathbf{1}, \mathbf{1 0}, \mathbf{1}, \mathbf{1}) \oplus(\mathbf{4}, \mathbf{1}, \mathbf{1}, \mathbf{2}) \oplus^{\oplus 2} \oplus(\mathbf{1}, \mathbf{1}, \mathbf{1}, \mathbf{1})$ \\
\hline $158 \mathfrak{g} \mathfrak{t}(4) \oplus \mathfrak{G} \mathfrak{i t}(8) \oplus \mathfrak{G} \mathfrak{t}(2)^{\oplus 2}$ & $(\overline{4}, 1,2,1) \oplus(1, \overline{8}, 2,1) \oplus(4,1,1,2) \oplus(1,8,1,2)$ \\
\hline $159 \mathfrak{g} \mathfrak{i t}(5) \oplus \mathfrak{S} \mathfrak{v}(10) \oplus \mathfrak{G} \mathfrak{t}(2)^{\oplus 2}$ & $(1,16,1,1) \oplus(\overline{5}, 1,2,1) \oplus(10,1,1,2) \oplus(1,11,1,1)^{\oplus 2}$ \\
\hline $160 \mathfrak{S} \mathfrak{i t}(5) \oplus \mathfrak{S} \mathfrak{v}(10) \oplus \mathfrak{G} \mathfrak{t}(2)^{\oplus 2}$ & $(1,16,1,1) \oplus(\overline{5}, 1,2,1) \oplus(10,1,1,2) \oplus(1,1,1,2)$ \\
\hline $161 \mathfrak{g} \mathfrak{u}(5) \oplus \mathfrak{S} \mathfrak{v}(10) \oplus \mathfrak{G} \mathfrak{t}(2)^{\oplus 2}$ & $(1,16,1,1) \oplus(\overline{5}, 1,2,1) \oplus(10,1,1,2) \oplus(1,1,2,1)$ \\
\hline $162 \mathfrak{g} \mathfrak{i t}(5) \oplus \mathfrak{s} \mathfrak{v}(10) \oplus \mathfrak{g} \mathfrak{t}(2)^{\oplus 2}$ & $(1,16,1,1) \oplus(\overline{5}, 1,2,1) \oplus(10,1,2,1) \oplus(1,1,1,2)$ \\
\hline $163 \mathfrak{S} \mathfrak{i t}(5) \oplus \mathfrak{S} \mathfrak{v}(10) \oplus \mathfrak{s} \mathfrak{t}(2)^{\oplus 2}$ & $(\overline{\mathbf{5}}, \mathbf{1}, \mathbf{1}, \mathbf{1})^{\oplus 2} \oplus(\mathbf{1}, \mathbf{1 6}, \mathbf{1}, \mathbf{1}) \oplus(\mathbf{1 0}, \mathbf{1}, \mathbf{2}, \mathbf{1}) \oplus(\mathbf{1}, \mathbf{1}, \mathbf{1}, \mathbf{2})$ \\
\hline $164 \mathfrak{S i t}(5) \oplus \mathfrak{S} \mathfrak{v}(10) \oplus \mathfrak{G} \mathfrak{t}(2)^{\oplus 2}$ & $(10,1,1,1)^{\oplus 2} \oplus(\mathbf{1}, \mathbf{1 6}, \mathbf{1}, \mathbf{1}) \oplus(\overline{5}, 1,2,1) \oplus(1,1,1,2)$ \\
\hline $165 \mathfrak{G H} \mathfrak{t}(5) \oplus \mathfrak{S} \mathfrak{u}(2)^{\oplus 3}$ & $(\overline{5}, 1,1,1) \oplus(\mathbf{1 0}, \mathbf{1}, \mathbf{1}, \mathbf{1}) \oplus(\overline{\mathbf{5}}, \mathbf{2}, \mathbf{1}, \mathbf{1}) \oplus(\mathbf{1 0}, \mathbf{1}, \mathbf{2}, \mathbf{1}) \oplus(\mathbf{1}, \mathbf{1}, \mathbf{1}, \mathbf{2}) \oplus(\mathbf{1}, \mathbf{1}, \mathbf{1}, \mathbf{1})$ \\
\hline $166 \mathfrak{g} \mathfrak{t}(5) \oplus \mathfrak{g} \mathfrak{t}(2)^{\oplus 3}$ & $(\overline{\mathbf{5}}, \mathbf{1}, \mathbf{1}, \mathbf{1}) \oplus(\mathbf{1 0}, \mathbf{1}, \mathbf{1}, \mathbf{1}) \oplus(\overline{\mathbf{5}}, \mathbf{2}, \mathbf{1}, \mathbf{1}) \oplus(\mathbf{1 0}, \mathbf{1}, \mathbf{2}, \mathbf{1}) \oplus(\mathbf{1}, \mathbf{1}, \mathbf{1}, 3)$ \\
\hline $167 \mathfrak{g} \mathfrak{i t}(5)^{\oplus 2} \oplus \mathfrak{G} \mathfrak{t}(2)^{\oplus 2}$ & $(\mathbf{1}, \overline{\mathbf{5}}, \mathbf{1}, \mathbf{1}) \oplus(\mathbf{1}, \mathbf{1 0}, \mathbf{1}, \mathbf{1}) \oplus(\overline{\mathbf{5}}, \mathbf{1}, \mathbf{2}, \mathbf{1}) \oplus(\mathbf{1 0}, \mathbf{1}, \mathbf{1}, \mathbf{2}) \oplus(\mathbf{1}, \mathbf{1}, \mathbf{1}, \mathbf{1})^{\oplus 3}$ \\
\hline $168 \mathfrak{G} \mathfrak{u}(5)^{\oplus 2} \oplus \mathfrak{G} \mathfrak{u}(2)^{\oplus 2}$ & $(\mathbf{1}, \overline{\mathbf{5}}, \mathbf{1}, \mathbf{1}) \oplus(\mathbf{1}, \mathbf{1 0}, \mathbf{1}, \mathbf{1}) \oplus(\overline{\mathbf{5}}, \mathbf{1}, \mathbf{2}, \mathbf{1}) \oplus(\mathbf{1 0}, \mathbf{1}, \mathbf{1}, \mathbf{2}) \oplus(\mathbf{1}, \mathbf{1}, \mathbf{1}, \mathbf{2}) \oplus(\mathbf{1}, \mathbf{1}, \mathbf{1}, \mathbf{1})$ \\
\hline $169 \mathfrak{G} \mathfrak{i t}(5)^{\oplus 2} \oplus \mathfrak{G} \mathfrak{t}(2)^{\oplus 2}$ & $(1, \overline{5}, 1,1) \oplus(1,10,1,1) \oplus(\overline{5}, 1,2,1) \oplus(10,1,1,2) \oplus(1,1,1,3)$ \\
\hline $170 \mathfrak{S} \mathfrak{i t}(5)^{\oplus 2} \oplus \mathfrak{G} \mathfrak{u t}(2)^{\oplus 2}$ & $(\mathbf{1}, \mathbf{5}, \mathbf{1}, \mathbf{1}) \oplus(\mathbf{1}, \mathbf{1 0}, \mathbf{1}, \mathbf{1}) \oplus(\overline{\mathbf{5}}, \mathbf{1}, \mathbf{2}, \mathbf{1}) \oplus(\mathbf{1 0}, \mathbf{1}, \mathbf{1}, \mathbf{2}) \oplus(\mathbf{1}, \mathbf{1}, \mathbf{2}, \mathbf{1}) \oplus(1,1,1,1)$ \\
\hline $171 \mathfrak{G} \mathfrak{u}(5)^{\oplus 2} \oplus \mathfrak{G} \mathfrak{u}(2)^{\oplus 2}$ & $(\mathbf{1}, \overline{\mathbf{5}}, \mathbf{1}, \mathbf{1}) \oplus(\mathbf{1}, \mathbf{1 0}, \mathbf{1}, \mathbf{1}) \oplus(\overline{\mathbf{5}}, \mathbf{1}, \mathbf{2}, \mathbf{1}) \oplus(\mathbf{1 0}, \mathbf{1}, \mathbf{1}, \mathbf{2}) \oplus(\mathbf{1}, \mathbf{1}, \mathbf{3}, \mathbf{1})$ \\
\hline $172 \mathfrak{S} \mathfrak{i t}(5)^{\oplus 2} \oplus \mathfrak{G} \mathfrak{t}(2)^{\oplus 2}$ & $(1, \overline{5}, 1,1) \oplus(1,10,1,1) \oplus(\overline{5}, 1,2,1) \oplus(10,1,2,1) \oplus(1,1,1,2) \oplus(1,1,1,1)$ \\
\hline $173 \mathfrak{S} \mathfrak{\mathfrak { t }}(5)^{\oplus 2} \oplus \mathfrak{G} \mathfrak{i t}(2)^{\oplus 2}$ & $(1, \overline{5}, 1,1) \oplus(1,10,1,1) \oplus(\overline{5}, 1,2,1) \oplus(10,1,2,1) \oplus(1,1,1,3)$ \\
\hline $174 \mathfrak{S} \mathfrak{i t}(5)^{\oplus 2} \oplus \mathfrak{G} \mathfrak{t}(2)^{\oplus 2}$ & $(\overline{\mathbf{5}}, \mathbf{1}, \mathbf{1}, \mathbf{1})^{\oplus} \oplus(\mathbf{1}, \overline{\mathbf{5}}, \mathbf{1}, \mathbf{1}) \oplus(\mathbf{1}, \mathbf{1 0}, \mathbf{1}, \mathbf{1}) \oplus(\mathbf{1 0}, \mathbf{1}, \mathbf{2}, \mathbf{1}) \oplus(\mathbf{1}, \mathbf{1}, \mathbf{1}, \mathbf{2}) \oplus(\mathbf{1}, \mathbf{1}, \mathbf{1}, \mathbf{1})$ \\
\hline $175 \mathfrak{S} \mathfrak{u}(5)^{\oplus 2} \oplus \mathfrak{G} \mathfrak{u}(2)^{\oplus 2}$ & $(\overline{\mathbf{5}}, \mathbf{1}, \mathbf{1}, \mathbf{1})^{\oplus} \oplus(\mathbf{1}, \overline{\mathbf{5}}, \mathbf{1}, \mathbf{1}) \oplus(\mathbf{1}, \mathbf{1 0}, \mathbf{1}, \mathbf{1}) \oplus(\mathbf{1 0}, \mathbf{1}, \mathbf{2}, \mathbf{1}) \oplus(\mathbf{1}, \mathbf{1}, \mathbf{1}, \mathbf{3})$ \\
\hline $176 \mathfrak{g} \mathfrak{u}(5)^{\oplus 2} \oplus \mathfrak{G} \mathfrak{t}(2)^{\oplus 2}$ & $(10,1,1,1)^{\oplus 2} \oplus(\mathbf{1}, \overline{\mathbf{5}}, \mathbf{1}, \mathbf{1}) \oplus(1,10,1,1) \oplus(\overline{\mathbf{5}}, 1,2,1) \oplus(1,1,1,2) \oplus(10,1,1,1)$ \\
\hline $177 \mathfrak{g} \mathfrak{i t}(5)^{\oplus 2} \oplus \mathfrak{G} \mathfrak{t}(2)^{\oplus 2}$ & $(10,1,1,1)^{\oplus 2} \oplus(\mathbf{1}, \overline{\mathbf{5}}, \mathbf{1}, \mathbf{1}) \oplus(\mathbf{1}, \mathbf{1 0}, \mathbf{1}, \mathbf{1}) \oplus(\overline{\mathbf{5}}, \mathbf{1}, \mathbf{2}, \mathbf{1}) \oplus(\mathbf{1}, \mathbf{1}, \mathbf{1}, \mathbf{3})$ \\
\hline $178 \mathfrak{g} \mathfrak{i t}(5)^{\oplus 3} \oplus \mathfrak{G} \mathfrak{i t}(2)$ & $\begin{array}{l}(\overline{\mathbf{5}}, \mathbf{1}, \mathbf{1}, \mathbf{1}) \oplus(\mathbf{1 0}, \mathbf{1}, \mathbf{1}, \mathbf{1}) \oplus(\mathbf{1}, \overline{\mathbf{5}}, \mathbf{1}, \mathbf{1}) \oplus(\mathbf{1}, \mathbf{1 0}, \mathbf{1}, \mathbf{1}) \oplus(\mathbf{1}, \mathbf{1}, \overline{\mathbf{5}}, \mathbf{1}) \oplus(\mathbf{1}, \mathbf{1}, \mathbf{1 0}, \mathbf{1}) \oplus \\
\quad(\mathbf{1}, \mathbf{1}, \mathbf{1}, \mathbf{2}) \oplus(\mathbf{1}, \mathbf{1}, \mathbf{1}, \mathbf{1})\end{array}$ \\
\hline $179 \mathfrak{S} \mathfrak{t}(5) \oplus \mathfrak{G} \mathfrak{t}(8) \oplus \mathfrak{g} \mathfrak{t}(2)^{\oplus 2}$ & $(\overline{5}, \mathbf{1}, \mathbf{1}, \mathbf{1}) \oplus(\mathbf{1 0}, \mathbf{1}, \mathbf{1}, \mathbf{1}) \oplus(\mathbf{1}, \overline{\mathbf{8}}, \mathbf{2}, \mathbf{1}) \oplus(\mathbf{1}, \mathbf{8}, \mathbf{1}, \mathbf{2}) \oplus(\mathbf{1}, \mathbf{1}, \mathbf{1}, \mathbf{1})$ \\
\hline $180 \mathfrak{g} \mathfrak{t}(4) \oplus \mathfrak{S} \mathfrak{v}(10)^{\oplus 2} \oplus \mathfrak{I} \mathfrak{u}(2)^{\oplus 2}$ & $(1,16,1,1,1) \oplus(1,1,16,1,1) \oplus(\overline{4}, 1,1,2,1) \oplus(4,1,1,1,2)$ \\
\hline $181 \mathfrak{G} \mathfrak{i t}(4) \oplus \mathfrak{g} \mathfrak{v}(10) \oplus \mathfrak{G} \mathfrak{t}(2)^{\oplus 3}$ & $(1,16,1,1,1) \oplus(4,1,2,2,1) \oplus(\overline{4}, 1,2,1,2)$ \\
\hline $182 \mathfrak{g} \mathfrak{i t}(4) \oplus \mathfrak{S} \mathfrak{v}(10) \oplus \mathfrak{G} \mathfrak{t}(2)^{\oplus 3}$ & $(\overline{4}, 1,2,1,1) \oplus(4,1,1,2,1) \oplus(1,16,1,1,2)$ \\
\hline $183 \mathfrak{S} \mathfrak{t}(4) \oplus \mathfrak{S} \mathfrak{v}(10) \oplus \mathfrak{G} \mathfrak{t}(2)^{\oplus 3}$ & $(1,16,1,1,1) \oplus(4,1,2,2,1) \oplus(\overline{4}, 1,1,1,2) \oplus 2$ \\
\hline $184 \mathfrak{g} \mathfrak{i t}(4) \oplus \mathfrak{S} \mathfrak{v}(10) \oplus \mathfrak{G} \mathfrak{t}(2)^{\oplus 3}$ & $(1,16,1,1,1) \oplus(4,1,2,2,1) \oplus(\overline{4}, 1,1,1,2) \oplus 2$ \\
\hline $185 \mathfrak{g} \mathfrak{i t}(4) \oplus \mathfrak{S} \mathfrak{v}(10) \oplus \mathfrak{G} \mathfrak{t}(2)^{\oplus 3}$ & $(1,16,1,1,1) \oplus(\overline{4}, 1,2,1,1) \oplus 2 \oplus(4,1,1,2,1) \oplus(4,1,1,1,2)$ \\
\hline $186 \mathfrak{g} \mathfrak{u t}(4) \oplus \mathfrak{s} \mathfrak{v}(10) \oplus \mathfrak{G} \mathfrak{t}(2)^{\oplus 3}$ & $(\underline{1}, 16,1,1,1) \oplus(\overline{4}, 1,2,1,1) \oplus 2 \oplus(4,1,1,2,1) \oplus(4,1,1,1,2)$ \\
\hline $187 \mathfrak{g} \mathfrak{i t}(4) \oplus \mathfrak{g} \mathfrak{p}(4) \oplus \mathfrak{I} \mathfrak{o}(10) \oplus \mathfrak{g} \mathfrak{i t}(2)^{\oplus 2}$ & $(\overline{4}, 4,1,1,1) \oplus(1,1,16,1,1) \oplus(4,1,1,2,1) \oplus(4,1,1,1,2)$ \\
\hline $188 \mathfrak{g} \mathfrak{u}(4) \oplus \mathfrak{g} \mathfrak{p}(4) \oplus \mathfrak{I} \mathfrak{o}(10) \oplus \mathfrak{g} \mathfrak{u}(2)^{\oplus 2}$ & $(\overline{4}, 4,1,1,1) \oplus(1,1,16,1,1) \oplus(4,1,1,2,1) \oplus(4,1,1,1,2)$ \\
\hline $189 \mathfrak{g} \mathfrak{t}(4) \oplus \mathfrak{g} \mathfrak{p}(4)^{\oplus 2} \oplus \mathfrak{G} \mathfrak{t}(2)^{\oplus 2}$ & $(\overline{4}, 4,1,1,1) \oplus(4,1,4,1,1) \oplus(\overline{4}, 1,1,2,1) \oplus(4,1,1,1,2)$ \\
\hline $190 \mathfrak{g} \mathfrak{t}(4) \oplus \mathfrak{S p}(4) \oplus \mathfrak{g} \mathfrak{t}(2)^{\oplus 3}$ & $(\overline{4}, 4,1,1,1) \oplus(4,1,3,2,1) \oplus(\underline{4}, 1,1,1,2)$ \\
\hline $191 \mathfrak{g} \mathfrak{t}(4) \oplus \mathfrak{S p}(4) \oplus \mathfrak{g} \mathfrak{t}(2)^{\oplus 3}$ & $(\overline{\mathbf{4}}, \mathbf{4}, \mathbf{1}, \mathbf{1}, \mathbf{1}) \oplus(\mathbf{4}, \mathbf{1}, 3,2,1) \oplus(\overline{4}, 1,1,1,2)$ \\
\hline $192 \mathfrak{G} \mathfrak{t}(4) \oplus \mathfrak{G p}(4) \oplus \mathfrak{G} \mathfrak{t}(2)^{\oplus 3}$ & $(\overline{4}, 4,1,1,1) \oplus(4,1,2,2,1) \oplus(4,1,2,1,1) \oplus(\overline{4}, 1,1,1,2)$ \\
\hline
\end{tabular}


TABLE II. (Continued)

Nonmaximal and Nonminimal algebras

\begin{tabular}{|c|c|c|}
\hline & Algebra & Fermion representations corresponding to $\beta$ \\
\hline 193 & $\mathfrak{S} \mathfrak{u}(4) \oplus \mathfrak{s} \mathfrak{p}(4) \oplus \mathfrak{s} \mathfrak{u}(2)^{\oplus 3}$ & $(\overline{4}, 4,1,1,1) \oplus(4,1,2,2,1) \oplus(4,1,2,1,1) \oplus(\overline{4}, 1,1,1,2)$ \\
\hline & $\mathfrak{S \mathfrak { u }}(4) \oplus \mathfrak{S} \mathfrak{p}(4) \oplus \mathfrak{s} \mathfrak{u}(2)^{\oplus 3}$ & $(\overline{4}, 4,1,1,1) \oplus(\overline{4}, 1,2,1,1) \oplus(4,1,1,2,1)^{\oplus 2} \oplus(4,1,1,1,2)$ \\
\hline & $\mathfrak{S} \mathfrak{u}(4) \oplus \mathfrak{S} \mathfrak{p}(4) \oplus \mathfrak{S} \mathfrak{u}(2)^{\oplus 3}$ & $(\overline{4}, 4,1,1,1) \oplus(\overline{4}, 1,2,1,1) \oplus(4,1,1,2,1)^{\oplus 2} \oplus(4,1,1,1,2)$ \\
\hline & $\mathfrak{g} \mathfrak{u}(4) \oplus \mathfrak{g} \mathfrak{p}(6) \oplus \mathfrak{g} \mathfrak{u}(2)^{\oplus 3}$ & $(\overline{4}, 6,1,1,1) \oplus(4,1,2,1,1) \oplus(4,1,1,2,1) \oplus(4,1,1,1,2)$ \\
\hline & $\mathfrak{S} \mathfrak{u}(4) \oplus \mathfrak{s} \mathfrak{p}(6) \oplus \mathfrak{s} \mathfrak{u}(2)^{\oplus 3}$ & $(\overline{4}, 6,1,1,1) \oplus(4,1,2,1,1) \oplus(4,1,1,2,1) \oplus(4,1,1,1,2)$ \\
\hline & $\mathfrak{S} \mathfrak{t}(4) \oplus \mathfrak{S} \mathfrak{u}(2)^{\oplus 4}$ & $(4,3,2,1,1) \oplus(\overline{4}, 1,1,3,2)$ \\
\hline & $\mathfrak{g} \mathfrak{u}(4) \oplus \mathfrak{g} \mathfrak{u}(2)^{\oplus 4}$ & $(4,2,2,1,1) \oplus(4,3,1,2,1) \oplus(4,1,1,1,2)$ \\
\hline & $\mathfrak{S} \mathfrak{u}(4) \oplus \mathfrak{s} \mathfrak{u}(2)^{\oplus 4}$ & $(4,2,2,1,1) \oplus(4,3,1,2,1) \oplus(4,1,1,1,2)$ \\
\hline & $\mathfrak{S} \mathfrak{u}(4) \oplus \mathfrak{s} \mathfrak{u}(2)^{\oplus 4}$ & $(4,2,2,1,1) \oplus(4,2,1,1,1) \oplus(\overline{4}, 1,1,3,2)$ \\
\hline & $\mathfrak{S} \mathfrak{u}(4) \oplus \mathfrak{g} \mathfrak{u}(2)^{\oplus 4}$ & $(4,2,2,1,1) \oplus(4,2,1,1,1) \oplus(\overline{4}, 1,1,3,2)$ \\
\hline & $\mathfrak{S} \mathfrak{u}(4) \oplus \mathfrak{g} \mathfrak{u}(2)^{\oplus 4}$ & $(4,2,2,1,1) \oplus(\overline{4}, 2,1,2,1) \oplus(4,1,2,1,1) \oplus(\overline{4}, 1,1,1,2)$ \\
\hline & $\mathfrak{S} \mathfrak{u}(4) \oplus \mathfrak{s} \mathfrak{u}(2)^{\oplus 4}$ & $(\mathbf{4}, \mathbf{2}, \mathbf{2}, \mathbf{1}, \mathbf{1}) \oplus(\overline{4}, \mathbf{2}, \mathbf{1}, \mathbf{2}, \mathbf{1}) \oplus(\mathbf{4}, \mathbf{1}, \mathbf{2}, \mathbf{1}, \mathbf{1}) \oplus(\overline{4}, \mathbf{1}, \mathbf{1}, \mathbf{1}, \mathbf{2})$ \\
\hline & $\mathfrak{s} \mathfrak{u}(4) \oplus \mathfrak{s} \mathfrak{u}(2)^{\oplus 4}$ & $(\mathbf{4}, \mathbf{2}, \mathbf{2}, \mathbf{1}, \mathbf{1}) \oplus(\mathbf{4}, \mathbf{2}, \mathbf{1}, \mathbf{1}, \mathbf{1}) \oplus(\overline{\mathbf{4}}, \mathbf{1}, \mathbf{1}, \mathbf{2}, \mathbf{2}) \oplus(\overline{\mathbf{4}}, \mathbf{1}, \mathbf{1}, \mathbf{2}, \mathbf{1})$ \\
\hline & $\mathfrak{S} \mathfrak{u}(4) \oplus \mathfrak{s} \mathfrak{u}(2)^{\oplus 4}$ & $(4,3,2,1,1) \oplus(\overline{4}, 1,1,2,1)^{\oplus 2} \oplus(\overline{4}, 1,1,1,2)$ \\
\hline & $\mathfrak{S} \mathfrak{u}(4) \oplus \mathfrak{s} \mathfrak{u}(2)^{\oplus 4}$ & $(4,3,2,1,1) \oplus(\overline{4}, 1,1,2,1) \oplus 2 \oplus(\overline{4}, 1,1,1,2)$ \\
\hline & $\mathfrak{S} \mathfrak{u}(4) \oplus \mathfrak{s} \mathfrak{u}(2)^{\oplus 4}$ & $(4,2,2,1,1) \oplus(\overline{4}, 1,1,2,1)^{\oplus 3} \oplus(4,1,1,1,2)$ \\
\hline & $\mathfrak{s} \mathfrak{u}(4) \oplus \mathfrak{s} \mathfrak{u}(2)^{\oplus 4}$ & $(4,2,2,1,1) \oplus(\overline{4}, 1,1,2,1))^{\oplus 3} \oplus_{-}(4,1,1,1,2)$ \\
\hline & $\mathfrak{S} \mathfrak{u}(4) \oplus \mathfrak{s} \mathfrak{u}(2)^{\oplus 4}$ & $(4,2,2,1,1) \oplus(4,2,1,1,1) \oplus(\overline{4}, 1,1,2,1)^{\oplus 2} \oplus(\overline{4}, 1,1,1,2)$ \\
\hline & $\mathfrak{S} \mathfrak{u}(4) \oplus \mathfrak{s} \mathfrak{u}(2)^{\oplus 4}$ & $(4,2,2,1,1) \oplus(4,2,1,1,1) \oplus(\overline{4}, 1,1,2,1)^{\oplus 2} \oplus(\overline{4}, 1,1,1,2)$ \\
\hline & $\mathfrak{G} \mathfrak{u}(4) \oplus \mathfrak{s} \mathfrak{u}(2)^{\oplus 4}$ & $(\overline{4}, \mathbf{2}, \mathbf{1}, \mathbf{1}, \mathbf{1})^{\oplus 2} \oplus(\overline{\mathbf{4}}, \mathbf{1}, \mathbf{2}, \mathbf{1}, \mathbf{1}) \oplus(\mathbf{4}, \mathbf{1}, \mathbf{1}, \mathbf{2}, \mathbf{1})^{\oplus 2} \oplus(\mathbf{4}, \mathbf{1}, \mathbf{1}, \mathbf{1}, \mathbf{2})$ \\
\hline & $\mathfrak{S} \mathfrak{u}(4) \oplus \mathfrak{s} \mathfrak{u}(2)^{\oplus 4}$ & $(\overline{4}, 2,1,1,1)^{\oplus 3} \oplus(4,1,2,1,1) \oplus(4,1,1,2,1) \oplus(4,1,1,1,2)$ \\
\hline & $\mathfrak{S} \mathfrak{u}(4) \oplus \mathfrak{g} \mathfrak{u}(2)^{\oplus 4}$ & $(\overline{4}, 2,1,1,1)^{\oplus 3} \oplus(4,1,2,1,1) \oplus(4,1,1,2,1) \oplus(4,1,1,1,2)$ \\
\hline & $\mathfrak{S} \mathfrak{u}(4)^{\oplus 2} \oplus \mathfrak{S} \mathfrak{v}(10) \oplus \mathfrak{S} \mathfrak{i t}(2)^{\oplus 2}$ & $(1,1,16,1,1) \oplus(4,1,1,2,1) \oplus(1,4,1,2,1) \oplus(4,1,1,1,2) \oplus(1,4,1,1,2)$ \\
\hline & $\mathfrak{S} \mathfrak{u}(4)^{\oplus 2} \oplus \mathfrak{S} \mathfrak{p}(4) \oplus \mathfrak{g} \mathfrak{t}(2)^{\oplus 2}$ & $(\overline{4}, 1,4,1,1) \oplus(4,1,1,2,1)^{\oplus 2} \oplus(1, \overline{4}, 1,2,1) \oplus(1,4,1,1,2)$ \\
\hline & $\mathfrak{G} \mathfrak{i t}(4)^{\oplus 2} \oplus \mathfrak{S} \mathfrak{p}(4) \oplus \mathfrak{G} \mathfrak{i t}(2)^{\oplus 2}$ & $(\overline{\mathbf{4}}, \mathbf{1}, \mathbf{4}, \mathbf{1}, \mathbf{1}) \oplus(\mathbf{4}, \mathbf{1}, \mathbf{1}, \mathbf{2}, \mathbf{1})^{\oplus 2} \oplus(\mathbf{1}, \overline{\mathbf{4}}, \mathbf{1}, \mathbf{2}, \mathbf{1}) \oplus(1,4,1,1,2)$ \\
\hline & $\mathfrak{S} \mathfrak{u}(4)^{\oplus 2} \oplus \mathfrak{g} \mathfrak{i}(2)^{\oplus 3}$ & $(\overline{4}, 6,1,1,1) \oplus(4,1,2,1,1) \oplus(4,1,1,2,1) \oplus(4,1,1,1,2)$ \\
\hline & $\mathfrak{S} \mathfrak{u}(4)^{\oplus 2} \oplus \mathfrak{S} \mathfrak{u}(2)^{\oplus 3}$ & $(4,1,2,2,1) \oplus(\overline{4}, 1,2,1,2) \oplus(1, \overline{4}, 1,2,1) \oplus(1,4,1,1,2)$ \\
\hline & $\mathfrak{s} \mathfrak{u}(4)^{\oplus 2} \oplus \mathfrak{G} \mathfrak{u}(2)^{\oplus 3}$ & $(\mathbf{4}, \mathbf{1}, \mathbf{2}, \mathbf{2}, \mathbf{1}) \oplus(\mathbf{1}, \overline{\mathbf{4}}, \mathbf{2}, \mathbf{1}, \mathbf{1}) \oplus(\overline{\mathbf{4}}, \mathbf{1}, \mathbf{1}, \mathbf{1}, \mathbf{2})^{\oplus 2} \oplus(\mathbf{1}, \mathbf{4}, \mathbf{1}, \mathbf{1}, \mathbf{2})$ \\
\hline & $\mathfrak{G} \mathfrak{u}(4)^{\oplus 2} \oplus \mathfrak{G} \mathfrak{u}(2)^{\oplus 3}$ & $(4,1,2,2,1) \oplus(1, \overline{4}, 2,1,1) \oplus(\overline{4}, 1,1,1,2)^{\oplus 2} \oplus(1,4,1,1,2)$ \\
\hline & $\mathfrak{S} \mathfrak{u}(4)^{\oplus 2} \oplus \mathfrak{g} \mathfrak{t}(2)^{\oplus 3}$ & $(\overline{4}, 1,2,1,1)^{\oplus 2} \oplus(1, \overline{4}, 2,1,1) \oplus(4,1,1,2,1) \oplus(1,4,1,2,1) \oplus(4,1,1,1,2)$ \\
\hline & $\mathfrak{S} \mathfrak{u}(4)^{\oplus 2} \oplus \mathfrak{G} \mathfrak{u}(2)^{\oplus 3}$ & $(\overline{4}, 1,2,1,1)^{\oplus 2} \oplus(1, \overline{4}, 2,1,1) \oplus(4,1,1,2,1) \oplus(1,4,1,2,1) \oplus(4,1,1,1,2)$ \\
\hline & $\mathfrak{s} \mathfrak{u}(4)^{\oplus 2} \oplus \mathfrak{s} \mathfrak{u}(2)^{\oplus 3}$ & $(\overline{4}, \mathbf{1}, \mathbf{2}, \mathbf{1}, \mathbf{1})^{\oplus 2} \oplus(\mathbf{1}, \overline{\mathbf{4}}, \mathbf{2}, \mathbf{1}, \mathbf{1}) \oplus(\mathbf{4}, \mathbf{1}, \mathbf{1}, \mathbf{2}, \mathbf{1})^{\oplus 2} \oplus(\mathbf{1}, \mathbf{4}, \mathbf{1}, \mathbf{1}, \mathbf{2})$ \\
\hline & $\mathfrak{G} \mathfrak{u}(4)^{\oplus 2} \oplus \mathfrak{G} \mathfrak{u}(2)^{\oplus 3}$ & $(\overline{4}, 1,2,1,1)^{\oplus 2} \oplus(1, \overline{4}, 2,1,1) \oplus(4,1,1,2,1)^{\oplus 2} \oplus(1,4,1,1,2)$ \\
\hline 226 & $\mathfrak{S} \mathfrak{u}(4)^{\oplus 2} \oplus \mathfrak{S} \mathfrak{u}(5) \oplus \mathfrak{S} \mathfrak{u}(2)^{\oplus 2}$ & $\begin{array}{l}(\mathbf{1}, \mathbf{1}, \mathbf{5}, \mathbf{1}, \mathbf{1}) \oplus(\mathbf{1}, \mathbf{1}, \mathbf{1 0}, \mathbf{1}, \mathbf{1}) \oplus(\overline{\mathbf{4}}, \mathbf{1}, \mathbf{1}, \mathbf{2}, \mathbf{1}) \oplus(\mathbf{1}, \overline{\mathbf{4}}, \mathbf{1}, \mathbf{2}, \mathbf{1}) \oplus(\mathbf{4}, \mathbf{1}, \mathbf{1}, \mathbf{1}, \mathbf{2}) \oplus \\
\quad(\mathbf{1}, \mathbf{4}, \mathbf{1}, \mathbf{1}, \mathbf{2}) \oplus(\mathbf{1}, \mathbf{1}, \mathbf{1}, \mathbf{1}, \mathbf{1})\end{array}$ \\
\hline 227 & $\mathfrak{S} \mathfrak{u}(4) \oplus \mathfrak{s} \mathfrak{u}(5) \oplus \mathfrak{S} \mathfrak{o}(10) \oplus \mathfrak{s} \mathfrak{u}(2)^{\oplus 2}$ & $\begin{array}{l}(\mathbf{1}, \overline{\mathbf{5}}, \mathbf{1}, \mathbf{1}, \mathbf{1}) \oplus(\mathbf{1}, \mathbf{1 0}, \mathbf{1}, \mathbf{1}, \mathbf{1}) \oplus(\mathbf{1}, \mathbf{1}, \mathbf{1 6}, \mathbf{1}, \mathbf{1}) \oplus(\overline{\mathbf{4}}, \mathbf{1}, \mathbf{1}, \mathbf{2}, \mathbf{1}) \oplus(\mathbf{4}, \mathbf{1}, \mathbf{1}, \mathbf{1}, \mathbf{2}) \oplus \\
\quad(\mathbf{1}, \mathbf{1}, \mathbf{1}, \mathbf{1}, \mathbf{1})\end{array}$ \\
\hline & $\mathfrak{S} \mathfrak{u}(4) \oplus \mathfrak{g} \mathfrak{u}(5) \oplus \mathfrak{s} \mathfrak{p}(4) \oplus \mathfrak{S} \mathfrak{u}(2)^{\oplus 2}$ & $(\overline{\mathbf{4}}, \mathbf{1}, \mathbf{4}, \mathbf{1}, \mathbf{1}) \oplus(\mathbf{1}, \overline{\mathbf{5}}, \mathbf{1}, \mathbf{1}, \mathbf{1}) \oplus(\mathbf{1}, \mathbf{1 0}, \mathbf{1}, \mathbf{1}, \mathbf{1}) \oplus(\mathbf{4}, \mathbf{1}, \mathbf{1}, \mathbf{2}, \mathbf{2}) \oplus(\mathbf{1}, \mathbf{1}, \mathbf{1}, \mathbf{1}, \mathbf{1})$ \\
\hline & $\mathfrak{S} \mathfrak{u}(4) \oplus \mathfrak{S} \mathfrak{u}(5) \oplus \mathfrak{S} \mathfrak{p}(4) \oplus \mathfrak{S} \mathfrak{u}(2)^{\oplus 2}$ & $(\overline{4}, 1,4,1,1) \oplus(1,5,1,1,1) \oplus(1,10,1,1,1) \oplus(4,1,1,2,2) \oplus(1,1,1,1,1)$ \\
\hline & $\mathfrak{S} \mathfrak{u}(4) \oplus \mathfrak{s} \mathfrak{u}(5) \oplus \mathfrak{s} \mathfrak{p}(4) \oplus \mathfrak{S} \mathfrak{u}(2)^{\oplus 2}$ & 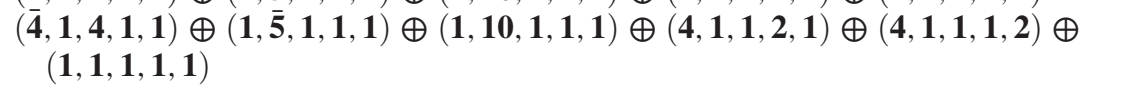 \\
\hline & $\mathfrak{S} \mathfrak{u}(4) \oplus \mathfrak{s} \mathfrak{u}(5) \oplus \mathfrak{s} \mathfrak{p}(4) \oplus \mathfrak{S} \mathfrak{u}(2)^{\oplus 2}$ & $\begin{array}{l}(\overline{\mathbf{4}}, \mathbf{1}, \mathbf{4}, \mathbf{1}, \mathbf{1}) \oplus(\mathbf{1}, \overline{\mathbf{5}}, \mathbf{1}, \mathbf{1}, \mathbf{1}) \oplus(\mathbf{1}, \mathbf{1 0}, \mathbf{1}, \mathbf{1}, \mathbf{1}) \oplus(\mathbf{4}, \mathbf{1}, \mathbf{1}, \mathbf{2}, \mathbf{1}) \oplus(\mathbf{4}, \mathbf{1}, \mathbf{1}, \mathbf{1}, \mathbf{2}) \oplus \\
\quad(\mathbf{1}, \mathbf{1}, \mathbf{1}, \mathbf{1}, \mathbf{1})\end{array}$ \\
\hline & $\mathfrak{S} \mathfrak{u t}(4) \oplus \mathfrak{S} \mathfrak{t}(5) \oplus \mathfrak{S} \mathfrak{u}(2)^{\oplus 3}$ & $(\mathbf{1}, \overline{\mathbf{5}}, \mathbf{1}, \mathbf{1}, \mathbf{1}) \oplus(\mathbf{1}, \mathbf{1 0}, \mathbf{1}, \mathbf{1}, \mathbf{1}) \oplus(\mathbf{4}, \mathbf{1}, \mathbf{2}, \mathbf{2}, \mathbf{1}) \oplus(\overline{\mathbf{4}}, \mathbf{1}, \mathbf{2}, \mathbf{1}, \mathbf{2}) \oplus(\mathbf{1}, \mathbf{1}, \mathbf{1}, \mathbf{1}, \mathbf{1})$ \\
\hline & $\mathfrak{g} \mathfrak{u}(4) \oplus \mathfrak{S} \mathfrak{t}(5) \oplus \mathfrak{g} \mathfrak{u}(2)^{\oplus 3}$ & $(\overline{4}, 1,2,1,1) \oplus(4,1,1,2,1) \oplus(1, \overline{5}, 1,1,2) \oplus(1,10,1,1,2) \oplus(1,1,1,1,1)^{\oplus 2}$ \\
\hline & $\mathfrak{S} \mathfrak{u}(4) \oplus \mathfrak{z} \mathfrak{u}(5) \oplus \mathfrak{s} \mathfrak{u}(2)^{\oplus 3}$ & $(1, \overline{5}, \mathbf{1}, \mathbf{1}, \mathbf{1})^{\oplus 2} \oplus(\overline{\mathbf{4}}, \mathbf{1}, \mathbf{2}, \mathbf{1}, \mathbf{1}) \oplus(\mathbf{4}, \mathbf{1}, \mathbf{1}, \mathbf{2}, \mathbf{1}) \oplus(\mathbf{1}, \mathbf{1 0}, \mathbf{1}, \mathbf{1}, \mathbf{2}) \oplus(\mathbf{1}, \mathbf{1}, \mathbf{1}, \mathbf{1}, \mathbf{1})^{\oplus 2}$ \\
\hline & $\mathfrak{S} \mathfrak{t}(4) \oplus \mathfrak{S} \mathfrak{t}(5) \oplus \mathfrak{g} \mathfrak{u}(2)^{\oplus 3}$ & $(1, \overline{5}, 1,1,1) \oplus(1,10,1,1,1) \oplus(4,1,2,2,1) \oplus(\overline{4}, 1,1,1,2)^{\oplus 2} \oplus(1,1,1,1,1)$ \\
\hline & $\mathfrak{g} \mathfrak{\mathfrak { t }}(4) \oplus \mathfrak{S} \mathfrak{t}(5) \oplus \mathfrak{g} \mathfrak{u}(2)^{\oplus 3}$ & $(1, \overline{\mathbf{5}}, \mathbf{1}, \mathbf{1}, \mathbf{1}) \oplus(1,10,1,1,1) \oplus(4,1,2,2,1) \oplus(\overline{\mathbf{4}}, 1,1,1,2)^{\oplus 2} \oplus(1,1,1,1,1)$ \\
\hline & $\mathfrak{g} \mathfrak{u}(4) \oplus \mathfrak{g} \mathfrak{u}(5) \oplus \mathfrak{g} \mathfrak{u}(2)^{\oplus 3}$ & $(1,10,1,1,1)^{\oplus 2} \oplus(\overline{4}, 1,2,1,1) \oplus(4,1,1,2,1) \oplus(1, \overline{5}, 1,1,2) \oplus(1,10,1,1,1)^{\oplus 2}$ \\
\hline & $\mathfrak{S} \mathfrak{u}(4) \oplus \mathfrak{S} \mathfrak{u}(5) \oplus \mathfrak{S} \mathfrak{u}(2)^{\oplus 3}$ & $(\overline{4}, 1,2,1,1) \oplus(4,1,1,2,1) \oplus(1, \overline{5}, 1,1,2) \oplus(1,10,1,1,2) \oplus(1,1,1,1,2)$ \\
\hline & $\mathfrak{s} \mathfrak{u}(4) \oplus \mathfrak{s} \mathfrak{t}(5) \oplus \mathfrak{s} \mathfrak{u}(2)^{\oplus 3}$ & $(1, \overline{5}, 1,1,1)^{\oplus 2} \oplus(\overline{4}, 1,2,1,1) \oplus(4,1,1,2,1) \oplus(1,10,1,1,2) \oplus(1,1,1,1,2)$ \\
\hline & $\mathfrak{S} \mathfrak{u}(4) \oplus \mathfrak{s} \mathfrak{u}(5) \oplus \mathfrak{s} \mathfrak{u}(2)^{\oplus 3}$ & 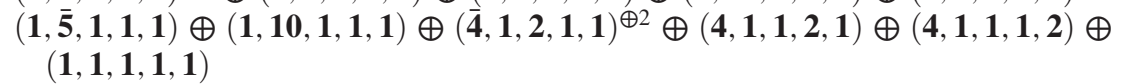 \\
\hline
\end{tabular}


TABLE II. (Continued)

Nonmaximal and Nonminimal algebras

\begin{tabular}{|c|c|}
\hline Algebra & Fermion representations corresponding to $\beta$ \\
\hline $241 \mathfrak{S} \mathfrak{u}(4) \oplus \mathfrak{S} \mathfrak{u}(5) \oplus \mathfrak{S} \mathfrak{u}(2)^{\oplus 3}$ & $\begin{array}{l}(\mathbf{1}, \overline{\mathbf{5}}, \mathbf{1}, \mathbf{1}, \mathbf{1}) \oplus(\mathbf{1}, \mathbf{1 0}, \mathbf{1}, \mathbf{1}, \mathbf{1}) \oplus(\overline{\mathbf{4}}, \mathbf{1}, \mathbf{2}, \mathbf{1}, \mathbf{1}) \oplus^{\oplus 2} \oplus(\mathbf{4}, \mathbf{1}, \mathbf{1}, \mathbf{2}, \mathbf{1}) \oplus(4,1,1,1,2) \oplus \\
\quad(\mathbf{1}, \mathbf{1}, \mathbf{1}, \mathbf{1}, \mathbf{1})\end{array}$ \\
\hline $242 \mathfrak{G} \mathfrak{t}(4) \oplus \mathfrak{G} \mathfrak{t}(5) \oplus \mathfrak{S} \mathfrak{t}(2)^{\oplus 3}$ & $(\mathbf{1}, \mathbf{1 0}, \mathbf{1}, \mathbf{1}, \mathbf{1})^{\oplus 2} \oplus(\overline{\mathbf{4}}, \mathbf{1}, \mathbf{2}, \mathbf{1}, \mathbf{1}) \oplus(\mathbf{4}, \mathbf{1}, \mathbf{1}, \mathbf{2}, \mathbf{1}) \oplus(\mathbf{1}, \overline{\mathbf{5}}, \mathbf{1}, \mathbf{1}, \mathbf{2}) \oplus(\mathbf{1}, \mathbf{1}, \mathbf{1}, \mathbf{1}, \mathbf{2})$ \\
\hline $243 \mathfrak{S} \mathfrak{i t}(4) \oplus \mathfrak{G} \mathfrak{t}(5) \oplus \mathfrak{S} \mathfrak{t}(2)^{\oplus 3}$ & $(1, \overline{\mathbf{5}}, \mathbf{1}, \mathbf{1}, \mathbf{1})^{\oplus 2} \oplus(\mathbf{1}, \mathbf{1 0}, \mathbf{1}, \mathbf{1}, \mathbf{1})^{\oplus 2} \oplus(\overline{\mathbf{4}}, \mathbf{1}, \mathbf{2}, \mathbf{1}, \mathbf{1}) \oplus(\mathbf{4}, \mathbf{1}, \mathbf{1}, \mathbf{2}, \mathbf{1}) \oplus(1,1,1,1,2)$ \\
\hline $244 \mathfrak{S} \mathfrak{u}(4) \oplus \mathfrak{S} \mathfrak{u}(5)^{\oplus 2} \oplus \mathfrak{G} \mathfrak{u}(2)^{\oplus 2}$ & $\begin{array}{l}(\mathbf{1}, \overline{\mathbf{5}}, \mathbf{1}, \mathbf{1}, \mathbf{1}) \oplus(\mathbf{1}, \mathbf{1 0}, \mathbf{1}, \mathbf{1}, \mathbf{1}) \oplus(1, \mathbf{1}, \overline{\mathbf{5}}, \mathbf{1}, \mathbf{1}) \oplus(\mathbf{1}, \mathbf{1}, \mathbf{1 0}, \mathbf{1}, \mathbf{1}) \oplus(\overline{\mathbf{4}}, \mathbf{1}, \mathbf{1}, \mathbf{2}, \mathbf{1}) \oplus \\
\quad(\mathbf{4}, \mathbf{1}, \mathbf{1}, \mathbf{1}, \mathbf{2}) \oplus(\mathbf{1}, \mathbf{1}, \mathbf{1}, \mathbf{1}, \mathbf{1}) \oplus 2\end{array}$ \\
\hline $245 \mathfrak{g} \mathfrak{u}(4) \oplus \mathfrak{S} \mathfrak{u}(8) \oplus \mathfrak{G} \mathfrak{u}(2)^{\oplus 3}$ & $(\overline{4}, 1,2,1,1) \oplus(1, \overline{8}, 2,1,1) \oplus(4,1,1,2,1) \oplus(1,8,1,1,2)$ \\
\hline $246 \mathfrak{S} \mathfrak{u t}(4) \oplus \mathfrak{G} \mathfrak{t}(8) \oplus \mathfrak{G} \mathfrak{t}(2)^{\oplus 3}$ & $(\overline{4}, 1,2,1,1) \oplus(1, \overline{8}, 2,1,1) \oplus(4,1,1,2,1) \oplus(1,8,1,1,2)$ \\
\hline $247 \mathfrak{g} \mathfrak{u}(4) \oplus \mathfrak{g} \mathfrak{v}(10) \oplus \mathfrak{G} \mathfrak{t}(2)^{\oplus 4}$ & $(1,16,1,1,1,1) \oplus(4,1,2,2,1,1) \oplus(\overline{4}, 1,1,1,2,1) \oplus(\overline{4}, 1,1,1,1,2)$ \\
\hline $248 \mathfrak{g} \mathfrak{u}(4) \oplus \mathfrak{g} \mathfrak{v}(10) \oplus \mathfrak{G} \mathfrak{t}(2)^{\oplus 4}$ & $(1,16,1,1,1,1) \oplus(4,1,2,2,1,1) \oplus(\overline{4}, 1,1,1,2,1) \oplus(\overline{4}, 1,1,1,1,2)$ \\
\hline $249 \mathfrak{g} \mathfrak{u}(4) \oplus \mathfrak{G} \mathfrak{v}(10) \oplus \mathfrak{g} \mathfrak{u}(2)^{\oplus 4}$ & $\begin{array}{l}(1,16,1,1,1,1) \oplus(\overline{4}, 1,2,1,1,1) \oplus(\overline{4}, 1,1,2,1,1) \oplus(4,1,1,1,2,1) \oplus \\
\quad(4,1,1,1,1,2)\end{array}$ \\
\hline $250 \quad \mathfrak{S} \mathfrak{t}(4) \oplus \mathfrak{s} \mathfrak{p}(4) \oplus \mathfrak{G} \mathfrak{t}(2)^{\oplus 4}$ & $(\overline{4}, 4,1,1,1,1) \oplus(4,1,2,2,1,1) \oplus(\overline{4}, 1,1,1,2,1) \oplus(4,1,1,1,1,2)$ \\
\hline $251 \mathfrak{g} \mathfrak{i t}(4) \oplus \mathfrak{s} \mathfrak{p}(4) \oplus \mathfrak{G} \mathfrak{t}(2)^{\oplus 4}$ & $(\overline{4}, 4,1,1,1,1) \oplus(4,1,2,2,1,1) \oplus(\overline{4}, 1,1,1,2,1) \oplus(4,1,1,1,1,2)$ \\
\hline $252 \mathfrak{S} \mathfrak{t}(4) \oplus \mathfrak{s} \mathfrak{p}(4) \oplus \mathfrak{S} \mathfrak{t}(2)^{\oplus 4}$ & $\begin{array}{l}(\overline{4}, \mathbf{4}, 1,1,1,1) \oplus(\overline{4}, 1,2,1,1,1) \oplus(4,1,1,2,1,1) \oplus(4,1,1,1,2,1) \oplus \\
\quad(4,1,1,1,1,2)\end{array}$ \\
\hline $253 \mathfrak{S} \mathfrak{t}(4) \oplus \mathfrak{s} \mathfrak{p}(4) \oplus \mathfrak{S} \mathfrak{t}(2)^{\oplus 4}$ & $\begin{array}{l}(\overline{4}, 4,1,1,1,1) \oplus(\overline{4}, 1,2,1,1,1) \oplus(4,1,1,2,1,1) \oplus(4,1,1,1,2,1) \oplus \\
\quad(4,1,1,1,1,2)\end{array}$ \\
\hline $254 \mathfrak{g} \mathfrak{t}(4) \oplus \mathfrak{G} \mathfrak{t}(2)^{\oplus 5}$ & $(4,2,2,1,1,1) \oplus(\overline{4}, 1,1,3,2,1) \oplus(4,1,1,1,1,2)$ \\
\hline $255 \mathfrak{g} \mathfrak{t}(4) \oplus \mathfrak{G} \mathfrak{i t}(2)^{\oplus 5}$ & $(4,2,2,1,1,1) \oplus(\overline{4}, 1,1,3,2,1) \oplus(4,1,1,1,1,2)$ \\
\hline $256 \mathfrak{g} \mathfrak{t}(4) \oplus \mathfrak{g} \mathfrak{t}(2)^{\oplus 5}$ & $(4,2,2,1,1,1) \oplus(\overline{4}, 2,1,2,1,1) \oplus(\overline{4}, 1,1,1,2,1) \oplus(4,1,1,1,1,2)$ \\
\hline $257 \mathfrak{g} \mathfrak{u}(4) \oplus \mathfrak{g} \mathfrak{t}(2)^{\oplus 5}$ & $(4,2,2,1,1,1) \oplus(4,2,1,1,1,1) \oplus(\underline{4}, 1,1,2,2,1) \oplus(\underline{4}, 1,1,1,1,2)$ \\
\hline $258 \mathfrak{g} \mathfrak{t}(4) \oplus \mathfrak{g} \mathfrak{t}(2)^{\oplus 5}$ & $(4,2,2,1,1,1) \oplus(4,2,1,1,1,1) \oplus(\overline{4}, 1,1,2,2,1) \oplus(\overline{4}, 1,1,1,1,2)$ \\
\hline $259 \mathfrak{G} \mathfrak{t}(4) \oplus \mathfrak{G} \mathfrak{t}(2)^{\oplus 5}$ & $(4,3,2,1,1,1) \oplus(\overline{4}, 1,1,2,1,1) \oplus(\overline{4}, 1,1,1,2,1) \oplus(\overline{4}, 1,1,1,1,2)$ \\
\hline $260 \mathfrak{g} \mathfrak{t}(4) \oplus \mathfrak{g} \mathfrak{t}(2)^{\oplus 5}$ & $(4,3,2,1,1,1) \oplus(\overline{4}, 1,1,2,1,1) \oplus(\overline{4}, 1,1,1,2,1) \oplus(\overline{4}, 1,1,1,1,2)$ \\
\hline $261 \mathfrak{g} \mathfrak{t}(4) \oplus \mathfrak{G} \mathfrak{u}(2)^{\oplus 5}$ & $(4,2,2,1,1,1) \oplus(\overline{4}, 1,1,2,1,1)^{\oplus 2} \oplus(\overline{4}, 1,1,1,2,1) \oplus(4,1,1,1,1,2)$ \\
\hline $262 \mathfrak{g} \mathfrak{u}(4) \oplus \mathfrak{g} \mathfrak{u}(2)^{\oplus 5}$ & $(4,2,2,1,1,1) \oplus(\overline{4}, 1,1,2,1,1)^{\oplus 2} \oplus_{-}(\overline{4}, 1,1,1,2,1) \oplus_{-}(4,1,1,1,1,2)$ \\
\hline $263 \mathfrak{s} \mathfrak{t}(4) \oplus \mathfrak{G} \mathfrak{u}(2)^{\oplus 5}$ & $\begin{array}{l}(\mathbf{4}, \mathbf{2}, 2,1,1,1) \oplus(4,2,1,1,1,1) \oplus(\overline{4}, 1,1,2,1,1) \oplus(\overline{4}, 1,1,1,2,1) \oplus \\
\quad(\overline{4}, 1,1,1,1,2)\end{array}$ \\
\hline $264 \mathfrak{g} \mathfrak{t}(4) \oplus \mathfrak{g} \mathfrak{i t}(2)^{\oplus 5}$ & $\begin{array}{l}(4,2,2,1,1,1) \oplus(4,2,1,1,1,1) \oplus(\overline{4}, 1,1,2,1,1) \oplus(\overline{4}, 1,1,1,2,1) \oplus \\
\quad(\overline{4}, 1,1,1,1,2)\end{array}$ \\
\hline $265 \mathfrak{g} \mathfrak{t}(4) \oplus \mathfrak{G} \mathfrak{i t}(2)^{\oplus 5}$ & $\begin{array}{l}(\overline{4}, 2,1,1,1,1)^{\oplus 2} \oplus(\overline{4}, 1,2,1,1,1) \oplus(4,1,1,2,1,1) \oplus(4,1,1,1,2,1) \\
\quad(4,1,1,1,1,2)\end{array}$ \\
\hline $266 \mathfrak{g} \mathfrak{t}(4) \oplus \mathfrak{g} \mathfrak{t}(2)^{\oplus 5}$ & $\begin{array}{l}(\overline{4}, 2,1,1,1,1)^{\oplus 2} \oplus(\overline{4}, 1,2,1,1,1) \oplus(4,1,1,2,1,1) \oplus(4,1,1,1,2,1) \oplus \\
\quad(\mathbf{4}, 1,1,1,1,2)\end{array}$ \\
\hline $267 \mathfrak{g} \mathfrak{t}(4)^{\oplus 2} \oplus \mathfrak{S} \mathfrak{o}(10) \oplus \mathfrak{I} \mathfrak{u}(2)^{\oplus 3}$ & $\begin{array}{l}(1,1,16,1,1,1) \oplus(\overline{4}, 1,1,2,1,1) \oplus(1, \overline{4}, 1,2,1,1) \oplus(4,1,1,1,2,1) \oplus \\
\quad(1,4,1,1,1,2)\end{array}$ \\
\hline $268 \mathfrak{g} \mathfrak{t}(4)^{\oplus 2} \oplus \mathfrak{I} \mathfrak{o}(10) \oplus \mathfrak{g} \mathfrak{u}(2)^{\oplus 3}$ & $\begin{array}{l}(1,1,16,1,1,1) \oplus(\overline{4}, 1,1,2,1,1) \oplus(1, \overline{4}, 1,2,1,1) \oplus(4,1,1,1,2,1) \oplus \\
\quad(1,4,1,1,1,2)\end{array}$ \\
\hline $269 \mathfrak{g} \mathfrak{t}(4)^{\oplus 2} \oplus \mathfrak{g} \mathfrak{p}(4) \oplus \mathfrak{g} \mathfrak{t}(2)^{\oplus 3}$ & $(\overline{4}, 1,4,1,1,1) \oplus(4,1,1,2,2,1) \oplus(1, \overline{4}, 1,2,1,1) \oplus(1,4,1,1,1,2)$ \\
\hline $270 \mathfrak{g} \mathfrak{i t}(4)^{\oplus 2} \oplus \mathfrak{g} \mathfrak{p}(4) \oplus \mathfrak{g} \mathfrak{t}(2)^{\oplus 3}$ & $(\overline{4}, 1,4,1,1,1) \oplus(4,1,1,2,2,1) \oplus(1, \overline{4}, 1,2,1,1) \oplus(1,4,1,1,1,2)$ \\
\hline $271 \mathfrak{S} \mathfrak{i t}(4)^{\oplus 2} \oplus \mathfrak{s} \mathfrak{p}(4) \oplus \mathfrak{G} \mathfrak{t}(2)^{\oplus 3}$ & $\begin{array}{l}(\overline{4}, 1,4,1,1,1) \oplus(4,1,1,2,1,1) \oplus(1, \overline{4}, 1,2,1,1) \oplus(4,1,1,1,2,1) \oplus \\
\quad(1,4,1,1,1,2)\end{array}$ \\
\hline $272 \mathfrak{G} \mathfrak{u}(4)^{\oplus 2} \oplus \mathfrak{g} \mathfrak{p}(4) \oplus \mathfrak{g} \mathfrak{u}(2)^{\oplus 3}$ & $\begin{array}{l}(\overline{\mathbf{4}}, \mathbf{1}, \mathbf{4}, \mathbf{1}, \mathbf{1}, \mathbf{1}) \oplus(4,1,1,2,1,1) \oplus(1, \overline{4}, 1,2,1,1) \oplus(4,1,1,1,2,1) \oplus \\
\quad(1,4,1,1,1,2)\end{array}$ \\
\hline $273 \mathfrak{s} \mathfrak{i t}(4)^{\oplus 2} \oplus \mathfrak{g} \mathfrak{p}(4) \oplus \mathfrak{g} \mathfrak{t}(2)^{\oplus 3}$ & $(\overline{\mathbf{4}}, \mathbf{1}, \mathbf{4}, \mathbf{1}, \mathbf{1}, \mathbf{1}) \oplus(\mathbf{4}, \mathbf{1}, \mathbf{1}, \mathbf{2}, \mathbf{1}, \mathbf{1})^{\oplus 2} \oplus(\mathbf{1}, \overline{\mathbf{4}}, \mathbf{1}, \mathbf{1}, \mathbf{2}, \mathbf{1}) \oplus(\mathbf{1}, \mathbf{4}, \mathbf{1}, \mathbf{1}, \mathbf{1}, \mathbf{2})$ \\
\hline $274 \mathfrak{g} \mathfrak{i t}(4)^{\oplus 2} \oplus \mathfrak{g} \mathfrak{p}(4) \oplus \mathfrak{g} \mathfrak{t}(2)^{\oplus 3}$ & $(\overline{4}, 1,4,1,1,1) \oplus(4,1,1,2,1,1)^{\oplus 2} \oplus(1, \overline{4}, 1,1,2,1) \oplus(1,4,1,1,1,2)$ \\
\hline $275 \mathfrak{g} \mathfrak{i t}(4)^{\oplus 2} \oplus \mathfrak{G i t}(2)^{\oplus 4}$ & $(4,1,2,2,1,1) \oplus(1, \overline{4}, 2,1,1,1) \oplus(\overline{4}, 1,1,1,2,2) \oplus(1,4,1,1,2,1)$ \\
\hline $276 \mathfrak{g} \mathfrak{u}(4)^{\oplus 2} \oplus \mathfrak{G} \mathfrak{t}(2)^{\oplus 4}$ & $(4,1,2,2,1,1) \oplus(\overline{4}, 1,2,1,2,1) \oplus(1, \overline{4}, 1,2,1,1) \oplus(1,4,1,1,1,2)$ \\
\hline $277 \mathfrak{g} \mathfrak{u}(4)^{\oplus 2} \oplus \mathfrak{G} \mathfrak{u}(2)^{\oplus 4}$ & $(4,1,2,2,1,1) \oplus(\overline{4}, 1,2,1,2,1) \oplus(1, \overline{4}, 1,2,1,1) \oplus(1,4,1,1,1,2)$ \\
\hline $278 \mathfrak{G} \mathfrak{u}(4)^{\oplus 2} \oplus \mathfrak{G} \mathfrak{u}(2)^{\oplus 4}$ & $\begin{array}{l}(\mathbf{4}, \mathbf{1}, 2,2,1,1) \oplus(1, \overline{4}, 2,1,1,1) \oplus(\overline{4}, 1,1,1,2,1) \oplus(1,4,1,1,2,1) \oplus \\
\quad(\overline{4}, 1,1,1,1,2)\end{array}$ \\
\hline $279 \mathfrak{g} \mathfrak{u}(4)^{\oplus 2} \oplus \mathfrak{G} \mathfrak{t}(2)^{\oplus 4}$ & 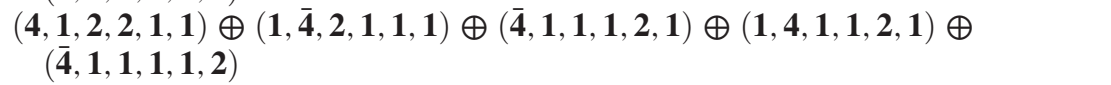 \\
\hline
\end{tabular}


TABLE II. (Continued)

Nonmaximal and Nonminimal algebras

\begin{tabular}{|c|c|}
\hline Algebra & Fermion representations corresponding to $\beta$ \\
\hline $280 \mathfrak{g} \mathfrak{t}(4)^{\oplus 2} \oplus \mathfrak{g} \mathfrak{t}(2)^{\oplus 4}$ & $(4,1,2,2,1,1) \oplus(1, \overline{4}, 2,1,1,1) \oplus(\overline{4}, 1,1,1,2,1)^{\oplus 2} \oplus(1,4,1,1,1,2)$ \\
\hline $281 \mathfrak{S} \mathfrak{u}(4)^{\oplus 2} \oplus \mathfrak{G} \mathfrak{u}(2)^{\oplus 4}$ & $(4,1,2,2,1,1) \oplus(1, \overline{4}, 2,1,1,1) \oplus(\overline{4}, 1,1,1,2,1)^{\oplus 2} \oplus(1,4,1,1,1,2)$ \\
\hline $282 \mathfrak{G} \mathfrak{i t}(4)^{\oplus 2} \oplus \mathfrak{G} \mathfrak{t}(2)^{\oplus 4}$ & 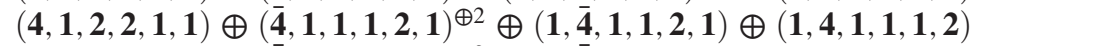 \\
\hline $283 \mathfrak{G} \mathfrak{u}(4)^{\oplus 2} \oplus \mathfrak{G} \mathfrak{t}(2)^{\oplus 4}$ & 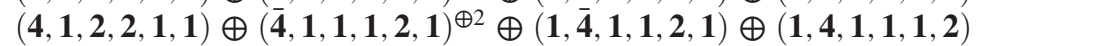 \\
\hline $284 \mathfrak{S} \mathfrak{u}(4)^{\oplus 2} \oplus \mathfrak{S} \mathfrak{u}(2)^{\oplus 4}$ & $\begin{array}{l}(\overline{4}, 1,2,1,1,1) \oplus(1, \overline{4}, 2,1,1,1) \oplus(\overline{4}, 1,1,2,1,1) \oplus(4,1,1,1,2,1) \oplus \\
\quad(1,4,1,1,2,1) \oplus(4,1,1,1,1,2)\end{array}$ \\
\hline $285 \mathfrak{g} \mathfrak{i t}(4)^{\oplus 2} \oplus \mathfrak{G} \mathfrak{t}(2)^{\oplus 4}$ & $\begin{array}{l}(\overline{4}, 1,2,1,1,1)^{\oplus 2} \oplus(1,4,2,1,1,1) \oplus(4,1,1,2,1,1) \oplus(4,1,1,1,2,1) \oplus \\
\quad(1,4,1,1,1,2)\end{array}$ \\
\hline $286 \mathfrak{g} \mathfrak{u}(4)^{\oplus 2} \oplus \mathfrak{g} \mathfrak{t}(2)^{\oplus 4}$ & $\begin{array}{l}(\overline{4}, 1,2,1,1,1)^{\oplus 2} \oplus(1, \overline{4}, 2,1,1,1) \oplus(4,1,1,2,1,1) \oplus(4,1,1,1,2,1) \oplus \\
\quad(1,4,1,1,1,2)\end{array}$ \\
\hline $287 \mathfrak{g} \mathfrak{t}(4)^{\oplus 2} \oplus \mathfrak{s} \mathfrak{t}(2)^{\oplus 4}$ & $\begin{array}{l}(\overline{4}, 1,2,1,1,1)^{\oplus 2} \oplus(4,1,1,2,1,1) \oplus(1, \overline{4}, 1,2,1,1) \oplus(4,1,1,1,2,1) \oplus \\
\quad(1,4,1,1,1,2)\end{array}$ \\
\hline $288 \mathfrak{g} \mathfrak{t}(4)^{\oplus 2} \oplus \mathfrak{g} \mathfrak{t}(2)^{\oplus 4}$ & $\begin{array}{l}(\overline{\mathbf{4}}, \mathbf{1}, \mathbf{2}, \mathbf{1}, \mathbf{1}, \mathbf{1})^{\oplus 2} \oplus(\mathbf{4}, \mathbf{1}, \mathbf{1}, \mathbf{2}, \mathbf{1}, \mathbf{1}) \oplus(\mathbf{1}, \overline{\mathbf{4}}, \mathbf{1}, \mathbf{2}, \mathbf{1}, \mathbf{1}) \oplus(\mathbf{4}, \mathbf{1}, \mathbf{1}, \mathbf{1}, \mathbf{2}, \mathbf{1}) \oplus \\
\quad(\mathbf{1}, \mathbf{4}, \mathbf{1}, \mathbf{1}, \mathbf{1}, \mathbf{2})\end{array}$ \\
\hline $289 \mathfrak{G} \mathfrak{i t}(4)^{\oplus 2} \oplus \mathfrak{s} \mathfrak{t}(2)^{\oplus 4}$ & $(\overline{\mathbf{4}}, \mathbf{1}, 2,1,1,1)^{\oplus 2} \oplus(\mathbf{4}, 1,1,2,1,1)^{\oplus 2} \oplus(\mathbf{1}, \overline{4}, 1,1,2,1) \oplus(1,4,1,1,1,2)$ \\
\hline $290 \mathfrak{g} \mathfrak{t}(4)^{\oplus 3} \oplus \mathfrak{S} \mathfrak{u}(2)^{\oplus 3}$ & $\begin{array}{l}(\overline{4}, 1,1,2,1,1) \oplus(1, \overline{4}, 1,2,1,1) \oplus(1,1, \overline{4}, 2,1,1) \oplus(4,1,1,1,2,1) \oplus \\
\quad(1,4,1,1,2,1) \oplus(1,1,4,1,1,2)\end{array}$ \\
\hline $291 \mathfrak{g} \mathfrak{t}(4)^{\oplus 3} \oplus \mathfrak{g} \mathfrak{t}(2)^{\oplus 3}$ & $\begin{array}{l}(\overline{4}, 1,1,2,1,1) \oplus(1, \overline{4}, 1,2,1,1) \oplus(1,1, \overline{4}, 2,1,1) \oplus(4,1,1,1,2,1) \oplus \\
\quad(1,4,1,1,2,1) \oplus(1,1,4,1,1,2)\end{array}$ \\
\hline $292 \mathfrak{g} \mathfrak{i t}(4)^{\oplus 2} \oplus \mathfrak{g} \mathfrak{t}(5) \oplus \mathfrak{g} \mathfrak{u}(2)^{\oplus 3}$ & $\begin{array}{l}(1,1, \overline{5}, 1,1,1) \oplus(1,1,10,1,1,1) \oplus(\overline{4}, 1,1,2,1,1) \oplus(1, \overline{4}, 1,2,1,1) \oplus \\
\quad(4,1,1,1,2,1) \oplus(1,4,1,1,1,2) \oplus(1,1,1,1,1,1)\end{array}$ \\
\hline $293 \mathfrak{g} \mathfrak{u}(4)^{\oplus 2} \oplus \mathfrak{g} \mathfrak{t}(5) \oplus \mathfrak{S} \mathfrak{u}(2)^{\oplus 3}$ & $\begin{array}{l}(1,1, \overline{5}, 1,1,1) \oplus(1,1,10,1,1,1) \oplus(\overline{4}, 1,1,2,1,1) \oplus(1, \overline{4}, 1,2,1,1) \oplus \\
\quad(4,1,1,1,2,1)\end{array}$ \\
\hline $294 \mathfrak{s} \mathfrak{t}(4) \oplus \mathfrak{s} \mathfrak{t}(5) \oplus \mathfrak{s} \mathfrak{t}(2)^{\oplus 4}$ & $\begin{array}{l}(1, \overline{\mathbf{5}}, \mathbf{1}, \mathbf{1}, \mathbf{1}, \mathbf{1}) \oplus(1,10,1,1,1,1) \oplus(4,1,2,2,1,1) \oplus(\overline{4}, 1,1,1,2,2) \oplus \\
\quad(\mathbf{1}, \mathbf{1}, \mathbf{1}, \mathbf{1}, \mathbf{1}, \mathbf{1})\end{array}$ \\
\hline $295 \mathfrak{s} \mathfrak{t}(4) \oplus \mathfrak{G} \mathfrak{t}(5) \oplus \mathfrak{s} \mathfrak{t}(2)^{\oplus 4}$ & $\begin{array}{l}(\overline{\mathbf{4}}, \mathbf{1}, \mathbf{2}, \mathbf{1}, \mathbf{1}, \mathbf{1}) \oplus(\mathbf{4}, \mathbf{1}, \mathbf{1}, \mathbf{2}, \mathbf{1}, \mathbf{1}) \oplus(\mathbf{1}, \overline{\mathbf{5}}, \mathbf{1}, \mathbf{1}, \mathbf{2}, \mathbf{1}) \oplus(\mathbf{1}, \mathbf{1 0}, \mathbf{1}, \mathbf{1}, \mathbf{1}, \mathbf{2}) \oplus \\
\quad(\mathbf{1}, \mathbf{1}, \mathbf{1}, \mathbf{1}, \mathbf{1}, \mathbf{1}) \oplus^{\oplus 2}\end{array}$ \\
\hline $296 \mathfrak{s} \mathfrak{t}(4) \oplus \mathfrak{s} \mathfrak{t}(5) \oplus \mathfrak{s} \mathfrak{t}(2)^{\oplus 4}$ & $\begin{array}{l}(\mathbf{1}, \overline{\mathbf{5}}, \mathbf{1}, \mathbf{1}, \mathbf{1}, \mathbf{1}) \oplus(\mathbf{1}, \mathbf{1 0}, \mathbf{1}, \mathbf{1}, \mathbf{1}, \mathbf{1}) \oplus(\mathbf{4}, \mathbf{1}, \mathbf{2}, 2, \mathbf{1}, \mathbf{1}) \oplus(\overline{\mathbf{4}}, \mathbf{1}, \mathbf{1}, \mathbf{1}, \mathbf{2}, \mathbf{1}) \oplus \\
\quad(\overline{\mathbf{4}}, \mathbf{1}, \mathbf{1}, \mathbf{1}, \mathbf{1}, \mathbf{2}) \oplus(\mathbf{1}, \mathbf{1}, \mathbf{1}, \mathbf{1}, \mathbf{1}, \mathbf{1})\end{array}$ \\
\hline $297 \mathfrak{g} \mathfrak{u}(4) \oplus \mathfrak{G} \mathfrak{t}(5) \oplus \mathfrak{g} \mathfrak{t}(2)^{\oplus 4}$ & $\begin{array}{l}(\mathbf{1}, \overline{\mathbf{5}}, \mathbf{1}, \mathbf{1}, \mathbf{1}, \mathbf{1}) \oplus(\mathbf{1}, \mathbf{1 0}, \mathbf{1}, \mathbf{1}, \mathbf{1}, \mathbf{1}) \oplus(\mathbf{4}, \mathbf{1}, \mathbf{2}, \mathbf{2}, \mathbf{1}, \mathbf{1}) \oplus(\overline{\mathbf{4}}, \mathbf{1}, \mathbf{1}, \mathbf{1}, \mathbf{2}, \mathbf{1}) \oplus \\
\quad(\overline{\mathbf{4}}, \mathbf{1}, \mathbf{1}, \mathbf{1}, \mathbf{1}, \mathbf{2}) \oplus(\mathbf{1}, \mathbf{1}, \mathbf{1}, \mathbf{1}, \mathbf{1}, \mathbf{1})\end{array}$ \\
\hline $298 \mathfrak{g} \mathfrak{t}(4) \oplus \mathfrak{s} \mathfrak{t}(5) \oplus \mathfrak{s} \mathfrak{t}(2)^{\oplus 4}$ & $\begin{array}{l}(\overline{4}, 1,2,1,1,1) \oplus(4,1,1,2,1,1) \oplus(1, \overline{5}, 1,1,2,1) \oplus(1,10,1,1,1,2) \oplus \\
\quad(1,1,1,1,1,2)\end{array}$ \\
\hline $299 \mathfrak{g} \mathfrak{t}(4) \oplus \mathfrak{s} \mathfrak{t}(5) \oplus \mathfrak{s} \mathfrak{t}(2)^{\oplus 4}$ & $\begin{array}{l}(\overline{4}, 1,2,1,1,1) \oplus(4,1,1,2,1,1) \oplus(1, \overline{5}, 1,1,2,1) \oplus(1,10,1,1,1,2) \oplus \\
\quad(1,1,1,1,2,1)\end{array}$ \\
\hline $300 \mathfrak{g} \mathfrak{t}(4) \oplus \mathfrak{G} \mathfrak{t}(5) \oplus \mathfrak{G} \mathfrak{t}(2)^{\oplus 4}$ & $\begin{array}{l}(\overline{4}, 1,2,1,1,1) \oplus(4,1,1,2,1,1) \oplus(1, \overline{5}, 1,1,2,1) \oplus(1,10,1,1,2,1) \oplus \\
\quad(1,1,1,1,1,2)\end{array}$ \\
\hline $301 \mathfrak{g} \mathfrak{t}(4) \oplus \mathfrak{s} \mathfrak{t}(5) \oplus \mathfrak{s} \mathfrak{t}(2)^{\oplus 4}$ & $\begin{array}{l}(\mathbf{1}, \overline{\mathbf{5}}, \mathbf{1}, \mathbf{1}, \mathbf{1}, \mathbf{1})^{\oplus 2} \oplus(\overline{\mathbf{4}}, \mathbf{1}, \mathbf{2}, \mathbf{1}, \mathbf{1}, \mathbf{1}) \oplus(\mathbf{4}, \mathbf{1}, \mathbf{1}, \mathbf{2}, \mathbf{1}, \mathbf{1}) \oplus(\mathbf{1}, \mathbf{1 0}, \mathbf{1}, \mathbf{1}, \mathbf{2}, \mathbf{1}) \oplus \\
\quad(\mathbf{1}, \mathbf{1}, \mathbf{1}, \mathbf{1}, \mathbf{1}, \mathbf{2})\end{array}$ \\
\hline $302 \mathfrak{g} \mathfrak{t}(4) \oplus \mathfrak{G} \mathfrak{t}(5) \oplus \mathfrak{G} \mathfrak{t}(2)^{\oplus 4}$ & $\begin{array}{l}(\mathbf{1}, \overline{\mathbf{5}}, \mathbf{1}, \mathbf{1}, \mathbf{1}, \mathbf{1}) \oplus(\mathbf{1}, \mathbf{1 0}, \mathbf{1}, \mathbf{1}, \mathbf{1}, \mathbf{1}) \oplus(\overline{\mathbf{4}}, \mathbf{1}, \mathbf{2}, \mathbf{1}, \mathbf{1}, \mathbf{1}) \oplus(\overline{\mathbf{4}}, \mathbf{1}, \mathbf{1}, \mathbf{2}, \mathbf{1}, \mathbf{1}) \oplus \\
\quad(\mathbf{4}, \mathbf{1}, \mathbf{1}, \mathbf{1}, \mathbf{2}, \mathbf{1}) \oplus(\mathbf{4}, \mathbf{1}, \mathbf{1}, \mathbf{1}, \mathbf{1}, \mathbf{2}) \oplus(\mathbf{1}, \mathbf{1}, \mathbf{1}, \mathbf{1}, \mathbf{1}, \mathbf{1})\end{array}$ \\
\hline $303 \mathfrak{s} \mathfrak{i t}(4) \oplus \mathfrak{G} \mathfrak{i}(5) \oplus \mathfrak{G} \mathfrak{u}(2)^{\oplus 4}$ & $\begin{array}{l}(1,10,1,1,1,1)^{\oplus 2} \oplus(\overline{4}, 1,2,1,1,1) \oplus(4,1,1,2,1,1) \oplus(1, \overline{\mathbf{5}}, \mathbf{1}, 1,2,1) \oplus \\
\quad(1,1,1,1,1,2)\end{array}$ \\
\hline $304 \mathfrak{g} \mathfrak{i t}(4) \oplus \mathfrak{S} \mathfrak{t}(5)^{\oplus 2} \oplus \mathfrak{g} \mathfrak{u}(2)^{\oplus 3}$ & $\begin{array}{l}(\mathbf{1}, \overline{\mathbf{5}}, \mathbf{1}, \mathbf{1}, \mathbf{1}, \mathbf{1}) \oplus(\mathbf{1}, \mathbf{1 0}, \mathbf{1}, \mathbf{1}, \mathbf{1}, \mathbf{1}) \oplus(\mathbf{1}, \mathbf{1}, \overline{\mathbf{5}}, \mathbf{1}, \mathbf{1}, \mathbf{1}) \oplus(\mathbf{1}, \mathbf{1}, \mathbf{1 0}, \mathbf{1}, \mathbf{1}, \mathbf{1}) \oplus \\
\quad(\overline{\mathbf{4}}, \mathbf{1}, \mathbf{1}, \mathbf{2}, \mathbf{1}, \mathbf{1}) \oplus(\mathbf{4}, \mathbf{1}, \mathbf{1}, \mathbf{1}, \mathbf{2}, \mathbf{1}) \oplus(\mathbf{1}, \mathbf{1}, \mathbf{1}, \mathbf{1}, \mathbf{1}, \mathbf{2})\end{array}$ \\
\hline $305 \mathfrak{g} \mathfrak{t}(4) \oplus \mathfrak{G} \mathfrak{t}(8) \oplus \mathfrak{s} \mathfrak{t}(2)^{\oplus 4}$ & $(\overline{4}, 1,2,1,1,1) \oplus(4,1,1,2,1,1) \oplus(1, \overline{8}, 1,1,2,1) \oplus(1,8,1,1,1,2)$ \\
\hline $306 \mathfrak{g} \mathfrak{u}(4) \oplus \mathfrak{g} \mathfrak{i t}(2)^{\oplus 6}$ & $(4,2,2,1,1,1,1) \oplus(\overline{4}, 1,1,2,2,1,1) \oplus(\overline{4}, 1,1,1,1,2,1) \oplus(4,1,1,1,1,1,2)$ \\
\hline $307 \mathfrak{g} \mathfrak{u}(4) \oplus \mathfrak{s} \mathfrak{i t}(2)^{\oplus 6}$ & $\begin{array}{l}(4,2,2,1,1,1,1) \oplus(\overline{4}, 1,1,2,1,1,1) \oplus(\overline{4}, 1,1,1,2,1,1) \oplus(\overline{4}, 1,1,1,1,2,1) \oplus \\
\quad(4,1,1,1,1,1,2)\end{array}$ \\
\hline $308 \mathfrak{g} \mathfrak{i t}(4) \oplus \mathfrak{g} \mathfrak{i t}(2)^{\oplus 6}$ & $\begin{array}{l}(4,2,2,1,1,1,1) \oplus(\overline{4}, 1,1,2,1,1,1) \oplus(\overline{4}, 1,1,1,2,1,1) \oplus(\overline{4}, 1,1,1,1,2,1) \oplus \\
\quad(4,1,1,1,1,1,2)\end{array}$ \\
\hline $309 \mathfrak{g} \mathfrak{u}(4) \oplus \mathfrak{g} \mathfrak{i t}(2)^{\oplus 6}$ & $\begin{array}{l}(\overline{4}, 2,1,1,1,1,1) \oplus(\overline{4}, 1,2,1,1,1,1) \oplus(\overline{4}, 1,1,2,1,1,1) \oplus(4,1,1,1,2,1,1) \oplus \\
\quad(4,1,1,1,1,2,1) \oplus(4,1,1,1,1,1,2)\end{array}$ \\
\hline
\end{tabular}


TABLE II. (Continued)

Nonmaximal and Nonminimal algebras

\begin{tabular}{|c|c|c|}
\hline & Algebra & Fermion representations corresponding to $\beta$ \\
\hline 310 & $\mathfrak{g} \mathfrak{i t}(4)^{\oplus 2} \oplus \mathfrak{g} \mathfrak{v}(10) \oplus \mathfrak{g} \mathfrak{t}(2)^{\oplus 4}$ & $\begin{array}{l}(1,1,16,1,1,1,1) \oplus(\overline{4}, 1,1,2,1,1,1) \oplus(4,1,1,1,2,1,1) \oplus(1, \overline{4}, 1,1,1,2,1) \oplus \\
\quad(1,4,1,1,1,1,2)\end{array}$ \\
\hline & $\mathfrak{G} \mathfrak{i t}(4)^{\oplus 2} \oplus \mathfrak{g} \mathfrak{p}(4) \oplus \mathfrak{G} \mathfrak{t}(2)^{\oplus 4}$ & $(\overline{4}, 1,4,1,1,1,1) \oplus(4,1,1,2,2,1,1) \oplus(1, \overline{4}, 1,1,1,2,1) \oplus(1,4,1,1,1,1,2)$ \\
\hline & $\mathfrak{S} \mathfrak{u}(4)^{\oplus 2} \oplus \mathfrak{g} \mathfrak{p}(4) \oplus \mathfrak{s} \mathfrak{t}(2)^{\oplus 4}$ & $(\overline{4}, 1,4,1,1,1,1) \oplus(4,1,1,2,2,1,1) \oplus(1, \overline{4}, 1,1,1,2,1) \oplus(1,4,1,1,1,1,2)$ \\
\hline & $\mathfrak{S} \mathfrak{i t}(4)^{\oplus 2} \oplus \mathfrak{I} \mathfrak{p}(4) \oplus \mathfrak{G} \mathfrak{t}(2)^{\oplus 4}$ & $\begin{array}{l}(\overline{4}, 1,4,1,1,1,1) \oplus(4,1,1,2,1,1,1) \oplus(4,1,1,1,2,1,1) \oplus(1, \overline{4}, 1,1,1,2,1) \oplus \\
\quad(1,4,1,1,1,1,2)\end{array}$ \\
\hline 314 & $\mathfrak{S} \mathfrak{u}(4)^{\oplus 2} \oplus \mathfrak{S} \mathfrak{p}(4) \oplus \mathfrak{G} \mathfrak{t}(2)^{\oplus 4}$ & $\begin{array}{l}(\overline{4}, 1,4,1,1,1,1) \oplus(4,1,1,2,1,1,1) \oplus(4,1,1,1,2,1,1) \oplus(1, \overline{4}, 1,1,1,2,1) \oplus \\
\quad(1,4,1,1,1,1,2)\end{array}$ \\
\hline & $\mathfrak{S} \mathfrak{u}(4)^{\oplus 2} \oplus \mathfrak{G} \mathfrak{u}(2)^{\oplus 5}$ & $(4,1,2,2,1,1,1) \oplus(1, \overline{4}, 2,1,1,1,1) \oplus(\overline{4}, 1,1,1,2,2,1) \oplus(1,4,1,1,1,1,2)$ \\
\hline & $\mathfrak{G} \mathfrak{u}(4)^{\oplus 2} \oplus \mathfrak{S} \mathfrak{u}(2)^{\oplus 5}$ & $(4,1,2,2,1,1,1) \oplus(1, \overline{4}, 2,1,1,1,1) \oplus(\overline{4}, 1,1,1,2,2,1) \oplus(1,4,1,1,1,1,2)$ \\
\hline 317 & $\mathfrak{S} \mathfrak{u}(4)^{\oplus 2} \oplus \mathfrak{G} \mathfrak{u}(2)^{\oplus 5}$ & $(4,1,2,2,1,1,1) \oplus(\overline{4}, 1,2,1,2,1,1) \oplus(1, \overline{4}, 1,1,1,2,1) \oplus(1,4,1,1,1,1,2)$ \\
\hline & $\mathfrak{S} \mathfrak{u t}(4)^{\oplus 2} \oplus \mathfrak{G} \mathfrak{u}(2)^{\oplus 5}$ & $\begin{array}{l}(4,1,2,2,1,1,1) \oplus(1, \overline{4}, 2,1,1,1,1) \oplus(\overline{4}, 1,1,1,2,1,1) \oplus(\overline{4}, 1,1,1,1,2,1) \oplus \\
\quad(1,4,1,1,1,1,2)\end{array}$ \\
\hline & $\mathfrak{S} \mathfrak{u}(4)^{\oplus 2} \oplus \mathfrak{G} \mathfrak{u t}(2)^{\oplus 5}$ & $\begin{array}{l}(4,1,2,2,1,1,1) \oplus(1, \overline{4}, 2,1,1,1,1) \oplus(\overline{4}, 1,1,1,2,1,1) \oplus(\overline{4}, 1,1,1,1,2,1) \oplus \\
\quad(1,4,1,1,1,1,2)\end{array}$ \\
\hline & $\mathfrak{S} \mathfrak{u}(4)^{\oplus 2} \oplus \mathfrak{S} \mathfrak{i t}(2)^{\oplus 5}$ & 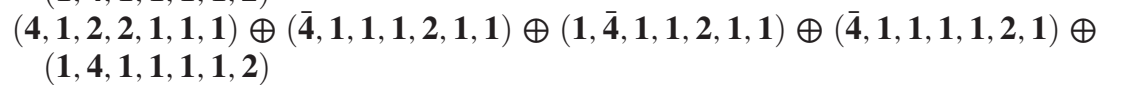 \\
\hline & $\mathfrak{S} \mathfrak{u}(4)^{\oplus 2} \oplus \mathfrak{G} \mathfrak{u}(2)^{\oplus 5}$ & $\begin{array}{l}(4,1,2,2,1,1,1) \oplus(\overline{4}, 1,1,1,2,1,1) \oplus(1, \overline{4}, 1,1,2,1,1) \oplus(\overline{4}, 1,1,1,1,2,1) \oplus \\
\quad(1,4,1,1,1,1,2)\end{array}$ \\
\hline & $\mathfrak{S} \mathfrak{i t}(4)^{\oplus 2} \oplus \mathfrak{S} \mathfrak{i t}(2)^{\oplus 5}$ & $(4,1,2,2,1,1,1) \oplus(\overline{4}, 1,1,1,2,1,1)^{\oplus 2} \oplus(1, \overline{4}, 1,1,1,2,1) \oplus(1,4,1,1,1,1,2)$ \\
\hline & $\mathfrak{G} \mathfrak{i t}(4)^{\oplus 2} \oplus \mathfrak{G} \mathfrak{i t}(2)^{\oplus 5}$ & $(4,1,2,2,1,1,1) \oplus(\overline{4}, 1,1,1,2,1,1)^{\oplus 2} \oplus(1, \overline{4}, 1,1,1,2,1) \oplus(1,4,1,1,1,1,2)$ \\
\hline & $\mathfrak{S} \mathfrak{u t}(4)^{\oplus 2} \oplus \mathfrak{S} \mathfrak{u}(2)^{\oplus 5}$ & $\begin{array}{l}(\overline{4}, 1,2,1,1,1,1) \oplus(1, \overline{4}, 2,1,1,1,1) \oplus(\overline{4}, 1,1,2,1,1,1) \oplus(4,1,1,1,2,1,1) \oplus \\
\quad(4,1,1,1,1,2,1) \oplus(1,4,1,1,1,1,2)\end{array}$ \\
\hline 325 & $\mathfrak{S} \mathfrak{u}(4)^{\oplus 2} \oplus \mathfrak{G} \mathfrak{u}(2)^{\oplus 5}$ & $\begin{array}{l}(\overline{4}, 1,2,1,1,1,1) \oplus(1, \overline{4}, 2,1,1,1,1) \oplus(\overline{4}, 1,1,2,1,1,1) \oplus(4,1,1,1,2,1,1) \oplus \\
\quad(4,1,1,1,1,2,1) \oplus(1,4,1,1,1,1,2)\end{array}$ \\
\hline 326 & $\mathfrak{S} \mathfrak{u t}(4)^{\oplus 2} \oplus \mathfrak{g} \mathfrak{u}(2)^{\oplus 5}$ & $\begin{array}{l}(\overline{\mathbf{4}}, \mathbf{1}, \mathbf{2}, \mathbf{1}, \mathbf{1}, \mathbf{1}, \mathbf{1})^{\oplus 2} \oplus(\mathbf{4}, \mathbf{1}, \mathbf{1}, \mathbf{2}, \mathbf{1}, \mathbf{1}, \mathbf{1}) \oplus(\mathbf{4}, \mathbf{1}, \mathbf{1}, \mathbf{1}, \mathbf{2}, \mathbf{1}, \mathbf{1}) \oplus(\mathbf{1}, \overline{\mathbf{4}}, \mathbf{1}, \mathbf{1}, \mathbf{1}, \mathbf{2}, \mathbf{1}) \oplus \\
\quad(\mathbf{1}, \mathbf{4}, \mathbf{1}, \mathbf{1}, \mathbf{1}, \mathbf{1}, \mathbf{2})\end{array}$ \\
\hline 327 & $\mathfrak{S} \mathfrak{u t}(4)^{\oplus 2} \oplus \mathfrak{s} \mathfrak{u}(2)^{\oplus 5}$ & $\begin{array}{l}(\overline{4}, \mathbf{1}, 2,1,1,1,1)^{\oplus 2} \\
\quad(1,4,1,1,1,1,2)\end{array}$ \\
\hline & $\mathfrak{S} \mathfrak{u}(4)^{\oplus 3} \oplus \mathfrak{g} \mathfrak{t}(2)^{\oplus 4}$ & $\begin{array}{l}(\overline{\mathbf{4}}, \mathbf{1}, \mathbf{1}, \mathbf{2}, 1,1,1) \oplus(1, \overline{\mathbf{4}}, 1,2,1,1,1) \oplus(1,1, \overline{4}, 2,1,1,1) \oplus(4,1,1,1,2,1,1) \oplus \\
\quad(1,4,1,1,1,2,1) \oplus(1,1,4,1,1,1,2)\end{array}$ \\
\hline & $\mathfrak{G} \mathfrak{u}(4)^{\oplus 3} \oplus \mathfrak{S} \mathfrak{t}(2)^{\oplus 4}$ & $\begin{array}{l}(\overline{4}, 1,1,2,1,1,1) \oplus(1,4,1,2,1,1,1) \oplus(1,1, \overline{4}, 2,1,1,1) \oplus(4,1,1,1,2,1,1) \oplus \\
\quad(1,4,1,1,1,2,1) \oplus(1,1,4,1,1,1,2)\end{array}$ \\
\hline & $\mathfrak{S} \mathfrak{i t}(4)^{\oplus 3} \oplus \mathfrak{g} \mathfrak{i t}(2)^{\oplus 4}$ & $\begin{array}{l}(\overline{4}, 1,1,2,1,1,1) \oplus(1, \overline{4}, 1,2,1,1,1) \oplus(4,1,1,1,2,1,1) \oplus(1,1, \overline{4}, 1,2,1,1) \oplus \\
\quad(1,4,1,1,1,2,1)\end{array}$ \\
\hline 331 & $\mathfrak{S} \mathfrak{u}(4)^{\oplus 3} \oplus \mathfrak{g} \mathfrak{u}(2)^{\oplus 4}$ & $\begin{array}{l}(\overline{4}, 1,1,2,1,1,1) \oplus(1, \overline{4}, 1,2,1,1,1) \oplus(4,1,1,1,2,1,1) \oplus(1,4,1,1,2,1,1) \oplus \\
\quad(1,1,4,1,1,2,1) \oplus(1,1,4,1,1,1,2)\end{array}$ \\
\hline 332 & $\mathfrak{S} \mathfrak{u}(4)^{\oplus 2} \oplus \mathfrak{I} \mathfrak{u}(5) \oplus \mathfrak{g} \mathfrak{u}(2)^{\oplus 4}$ & $\begin{array}{l}(1, \mathbf{1}, \overline{\mathbf{5}}, \mathbf{1}, \mathbf{1}, \mathbf{1}, \mathbf{1}) \oplus(1,1,10,1,1,1,1) \oplus(\overline{4}, 1,1,2,1,1,1) \oplus(4,1,1,1,2,1,1) \oplus \\
\quad(1, \overline{4}, 1,1,1,2,1) \oplus(1,4,1,1,1,1,2) \oplus(1,1,1,1,1,1,1)\end{array}$ \\
\hline 333 & $\mathfrak{S} \mathfrak{i t}(4) \oplus \mathfrak{S} \mathfrak{i t}(5) \oplus \mathfrak{g} \mathfrak{i t}(2)^{\oplus 5}$ & $\begin{array}{l}(\overline{4}, 1,2,1,1,1,1) \oplus(4,1,1,2,1,1,1) \oplus(1, \overline{5}, 1,1,2,1,1) \oplus(1,10,1,1,1,2,1) \oplus \\
\quad(1,1,1,1,1,1,2)\end{array}$ \\
\hline 334 & $\mathfrak{S} \mathfrak{u t}(4)^{\oplus 2} \oplus \mathfrak{s} \mathfrak{u}(2)^{\oplus 6}$ & $\begin{array}{l}(\mathbf{4}, \mathbf{1}, 2,2,1,1,1,1) \oplus(\overline{4}, 1,1,1,2,2,1,1) \oplus(1, \overline{4}, 1,1,1,1,2,1) \oplus \\
\quad(1,4,1,1,1,1,1,2)\end{array}$ \\
\hline 335 & $\mathfrak{S} \mathfrak{u}(4)^{\oplus 2} \oplus \mathfrak{g} \mathfrak{u}(2)^{\oplus 6}$ & $\begin{array}{l}(4,1,2,2,1,1,1,1) \oplus(\overline{4}, 1,1,1,2,1,1,1) \oplus(\overline{4}, 1,1,1,1,2,1,1) \oplus \\
\quad(1, \overline{4}, 1,1,1,1,2,1) \oplus(1,4,1,1,1,1,1,2)\end{array}$ \\
\hline 336 & $\mathfrak{S} \mathfrak{u t}(4)^{\oplus 2} \oplus \mathfrak{s} \mathfrak{u}(2)^{\oplus 6}$ & $\begin{array}{l}(4, \mathbf{1}, \mathbf{2}, \mathbf{2}, \mathbf{1}, \mathbf{1}, \mathbf{1}, \mathbf{1}) \oplus(\overline{\mathbf{4}}, \mathbf{1}, \mathbf{1}, \mathbf{1}, \mathbf{2}, \mathbf{1}, \mathbf{1}, \mathbf{1}) \oplus(\overline{4}, \mathbf{1}, \mathbf{1}, \mathbf{1}, \mathbf{1}, \mathbf{2}, \mathbf{1}, \mathbf{1}) \oplus \\
\quad(\mathbf{1}, \overline{\mathbf{4}}, \mathbf{1}, \mathbf{1}, \mathbf{1}, \mathbf{1}, \mathbf{2}, \mathbf{1}) \oplus(\mathbf{1}, \mathbf{4}, \mathbf{1}, \mathbf{1}, \mathbf{1}, \mathbf{1}, \mathbf{1}, \mathbf{2})\end{array}$ \\
\hline 337 & $\mathfrak{S} \mathfrak{i t}(4)^{\oplus 2} \oplus \mathfrak{S} \mathfrak{i t}(2)^{\oplus 6}$ & $\begin{array}{l}(\overline{4}, 1,2,1,1,1,1,1) \oplus(\overline{4}, 1,1,2,1,1,1,1) \oplus(4,1,1,1,2,1,1,1) \oplus \\
\quad(4,1,1,1,1,2,1,1) \oplus(1, \overline{4}, 1,1,1,1,2,1) \oplus(1,4,1,1,1,1,1,2)\end{array}$ \\
\hline & $\mathfrak{S} \mathfrak{u}(4)^{\oplus 3} \oplus \mathfrak{g} \mathfrak{u}(2)^{\oplus 5}$ & $\begin{array}{l}(\overline{4}, 1,1,2,1,1,1,1) \oplus(1, \overline{4}, 1,2,1,1,1,1) \oplus(4,1,1,1,2,1,1,1) \oplus \\
\quad(1,4,1,1,1,2,1,1) \oplus(1,1, \overline{4}, 1,1,1,2,1) \oplus(1,1,4,1,1,1,1,2)\end{array}$ \\
\hline & $\mathfrak{G} \mathfrak{u}(4)^{\oplus 3} \oplus \mathfrak{g} \mathfrak{t}(2)^{\oplus 5}$ & $\begin{array}{l}(\overline{4}, 1,1,2,1,1,1,1) \oplus(1, \overline{4}, 1,2,1,1,1,1) \oplus(4,1,1,1,2,1,1,1) \oplus \\
\quad(1,4,1,1,1,2,1,1) \oplus(1,1,4,1,1,1,2,1) \oplus(1,1,4,1,1,1,1,2)\end{array}$ \\
\hline & $\mathfrak{S} \mathfrak{u}(4)^{\oplus 3} \oplus \mathfrak{g} \mathfrak{u}(2)^{\oplus 6}$ & $\begin{array}{r}(\overline{4}, 1,1,2,1,1,1,1,1) \oplus(4,1,1,1,2,1,1,1,1) \oplus(1,4,1,1,1,2,1,1,1) \oplus \\
\quad(1,4,1,1,1,1,2,1,1) \oplus(1,1,4,1,1,1,1,2,1) \oplus(1,1,4,1,1,1,1,1,2)\end{array}$ \\
\hline
\end{tabular}


isomorphism (i.e., if $j$ also surjects). We say such a diagram (1) is maximal (respectively, minimal) if the only morphisms out of (respectively, into) it are equivalences.

Our goal is then to find all inequivalent diagrams $(\mathfrak{g}, \alpha, \beta, \gamma)$ for which $\gamma$ corresponds to the SM embedding. To do so, we choose Cartan subalgebras of each algebra appearing in diagram (1), which we denote $\mathfrak{h}_{\mathfrak{s} \mathfrak{m}}, \mathfrak{h}$, and $\mathfrak{h}_{48}$ for $\mathfrak{s m}, \mathfrak{g}$, and $\mathfrak{s} \mathfrak{u}(48)$, respectively. Up to equivalence, we choose $\alpha, \beta$, and $\gamma$ such that these get embedded into one another (along with a compatible restriction on morphisms).

Equivalently, using the universal property of the direct sum, we seek a pair of diagrams

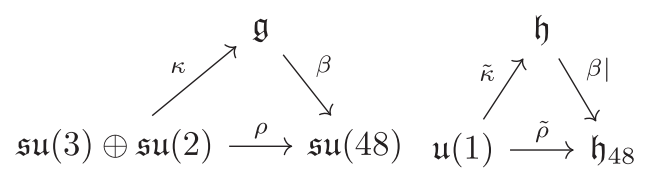

where $\gamma=\rho \oplus \tilde{\rho}$ corresponds to the chosen SM embedding, $\alpha=\kappa \oplus \tilde{\kappa}$, and $\beta \mid$ on the right-hand side denotes the obvious restriction map.

\section{COMPUTATION}

In rough terms, our approach to the computation is as follows. The first step is to evaluate all embeddings $\beta$ in Eq. (1). Up to inner automorphisms of $\mathfrak{g} \mathfrak{t}(48)$ these are inequivalent representations of semisimple Lie algebras of dimension 48 and so can be found using standard representation theory techniques. We keep only those which are anomaly free. For every $\mathfrak{g}$ for which a $\beta$ exists, we then find all embeddings $\kappa$ up to outer automorphisms using the theory of maximal embeddings. We then find all $\kappa$ and $\beta$ such that there exists a $\rho$ matching the embedding of the SM up to inner automorphisms, as in the left-hand diagram in Eq. (3). For a given diagram, we then determine if compatible $\tilde{\kappa}$ and $\tilde{\rho}$ exist (taking account of possible inner automorphisms which may need to be applied). By finding all embeddings $j: \mathfrak{g}^{\prime} \rightarrow \mathfrak{g}$ for algebras which appear in our final list, our program then checks which are maximal and which are minimal.

The program itself uses projection matrices rather than embeddings. Projection matrices describe how the weights project from the superalgebra to the subalgebra. For a given $\mathfrak{g}$, the projection matrices corresponding to potentially allowed $\kappa$ 's can be found using the theory of maximal embeddings [7-11]. (We use the Mathematica program LieART2.0 $[12,13]$ to generate the maximal projection matrices themselves). One has to take care to ensure that these projection matrices lift correctly to embeddings, and to ensure that they define a unique diagram [Eq. (1)] up to equivalence.

The output of the program (provided in Supplemental Matrials [6]) consists of the highest weights of the representation specified by $\beta$ and the projection matrix of the embedding $\kappa$, to which we have appended a final row specifying $\tilde{\kappa}$ [to wit, acting on the weights of $\mathfrak{g}$, this row returns the corresponding $\mathfrak{t}(1) \subset \mathfrak{g m}$ charges].

This approach results in a number of practical issues when it comes to carrying out the computation, which we now describe, along with their resolutions, in rough order of importance.

(i) Since $\mathfrak{g} \mathfrak{u}(2)$ has anomaly-free irreducible representations of every dimension, there are many possible anomaly-free embeddings of ideals of $\mathfrak{g}$ made up of $\mathfrak{S} \mathfrak{t}(2) \mathrm{s}$ in $\mathfrak{s} \mathfrak{t}(48)$. For example, there are $\mathcal{O}\left(10^{5}\right)$ for $\mathfrak{g}=$ $\mathfrak{g} \mathfrak{t}(2)$ and $\mathcal{O}\left(10^{7}\right)$ for $\mathfrak{g}=\mathfrak{g} \mathfrak{t}(2)^{\oplus 2}$. We reduce this by first finding all possible $\beta$ for $\mathfrak{g}$ without an $\mathfrak{g} \mathfrak{t}(2)$ ideal and then requiring that they contain $\mathfrak{s \mathfrak { t }}(3) \subset \mathfrak{s m}$ [here we use the fact that the restriction of $\kappa$ to $\mathfrak{g} \mathfrak{t}(3)$ has to map trivially into any $\mathfrak{g} \mathfrak{u}(2)$ ideal of $\mathfrak{g}$ ]. To these $\mathfrak{g}$ we add all possible ideals made up of $\mathfrak{g} \mathfrak{t}(2)$ 's and retest to see if a full $\kappa$ exists.

(ii) Even after ignoring ideals made up of $\mathfrak{g} \mathfrak{t}(2)$ 's, there are still $\mathcal{O}\left(10^{6}\right)$ anomaly-free embeddings of $\mathfrak{g}$. We determine these in a bottom-up fashion by first finding the $\mathcal{O}\left(10^{3}\right)$ anomaly-free representations of dimension 48 of the $\mathcal{O}\left(10^{2}\right)$ possible simple $\mathfrak{g}$ [e.g., $\mathbf{5} \oplus \overline{\mathbf{1 0}}$ plus 33 singlets of $\mathfrak{g} \mathfrak{u}(5)]$ and then using these to find all anomalyfree representations of possible semisimple $\mathfrak{g}$ of dimension 48. Here, we use the fact that a representation of a semisimple algebra is anomaly-free iff its restriction to any simple ideal is anomaly free. For example, the representations of $\mathfrak{g} \mathfrak{t}(4) \oplus \mathfrak{a p}(4)$ given by

(i) $(\mathbf{4}, \mathbf{4}) \oplus(\overline{\mathbf{4}}, \mathbf{1})^{\oplus 4} \oplus(\mathbf{1}, \mathbf{1})^{\oplus 16}$

(ii) $(\overline{\mathbf{4}}, \mathbf{4}) \oplus(\mathbf{4}, \mathbf{1})^{\oplus 4} \oplus(\mathbf{1}, \mathbf{1})^{\oplus 16}$

(iii) $(\mathbf{4}, \mathbf{1})^{\oplus 4} \oplus(\overline{\mathbf{4}}, \mathbf{1})^{\oplus 4} \oplus(\mathbf{1}, \mathbf{4})^{\oplus 4}$

are anomaly free because they all reduce to the $\mathbf{4}^{\oplus 4} \oplus \overline{\mathbf{4}}^{\oplus 4}$ of $\mathfrak{g} \mathfrak{u}(4)$ and the $\mathbf{4}^{\oplus 4}$ of $\mathfrak{s p}(4)$ (plus the appropriate number of singlets). This bottom-up method is also used later to find all representations when ideals made up of $\mathfrak{s} \mathfrak{u}(2)$ 's are included.

(iii) Finding the possible representations of semisimple algebras above requires considering a large number of permutations of a list (as do other steps in the calculation, e.g., finding $\kappa$ from maximal embeddings). For example, to find the anomaly-free representations $1-3$ above requires consideration of around 500 different permutations. The computation is greatly expedited by the use of an algorithm that determines which permutations can be skipped based on previous cases.

Two more minor improvements are as follows: (iv) the fact that one can discard $\beta$ whose corresponding representation has a nontrivial part of dimension fewer than 45 [or 36 before we include $\mathfrak{g} \mathfrak{t}(2)$ ideals], since these cannot lead to a valid $\alpha$; (v) in a similar vein, no $\alpha$ exists for those $\beta$ whose corresponding representations have more than three vanishing weights, or weights in negative pairs, since such weights must be associated with $\mathfrak{g m}$ singlets of which there are just three. 
The program took less than an hour to run on a personal computer. As such, model builders should find it easy to adapt it to other cases of interest. ${ }^{4}$

\section{CLOSING REMARKS}

We have produced, for the first time, a definitive list of semisimple Lie algebras that contain the SM Lie algebra, are free of local anomalies, and act by a unitary representation on SM fermions plus three singlet neutrinos. Such extensions can mix flavor, color, and electroweak symmetries in nontrivial ways. Table I shows the 26 maximal algebras and six minimal ones, whereas the remainder are provided in Table II. In total, there are 340 physically inequivalent algebras. No exceptional Lie algebras appear, since they require fermions beyond those in the SM plus three singlet neutrinos. Many of the symmetries listed are semifamiliar, being variations on the theme of well-known unification and flavor symmetries. A few of the symmetries in our catalog have the novel feature of combining unification and flavor symmetries in an essential way, motivating their further study. For example, we have

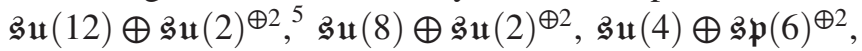
$\mathfrak{s} \mathfrak{u}(4) \oplus \mathfrak{g} \mathfrak{p}(6) \oplus \mathfrak{g} \mathfrak{p}(6)$, and $\mathfrak{g} \mathfrak{u}(4) \oplus \mathfrak{s} \mathfrak{p}(4)^{\oplus 2}$.

By concentrating upon semisimple algebras, we have neglected the class of models with Abelian summands in their gauge Lie algebras. Previously, a few very particular

\footnotetext{
${ }^{4}$ The programs and instructions on their use can be found in the ancillary information attached to the arXiv preprint version of the present paper.
}

cases with additional summands of $\mathfrak{t}(1)$ have had their anomaly cancellation conditions solved with quite some effort [1,15-19] but there are currently no known methods for a fixed matter field content, but general Lie algebra. Including such Abelian summands then implies a significant increase in difficulty as compared to not including them. One could imagine having to find particular geometric constructions for each possibility of the semisimple algebra. In order to sidestep this difficulty, we have narrowed the scope to examine only semisimple extensions. This, however, implicitly includes all cases where an Abelian summand is ultimately derived from a semisimple one through its spontaneous breaking, which could happen at an intermediate stage between $\mathfrak{g}$ and $\mathfrak{s m}$.

Adding additional matter fields changes the list, but is straightforward to carry out, in principle. An interesting example to investigate would be to add a Dirac fermion in the same representation as the Higgs boson, since it constitutes a viable candidate for weakly interacting thermal relic dark matter.

\section{ACKNOWLEDGMENTS}

We thank J. Davighi and other members of the Cambridge Pheno Working Group for discussions. This work was supported by Science and Technology Facilities Council consolidated Grants No. ST/P000681/1, No. ST/ T000694/1, and No. ST/S505316/1.

\footnotetext{
${ }^{5}$ The $\mathfrak{g} \mathfrak{t}(12) \oplus \mathfrak{g} \mathfrak{t}(2) \oplus \mathfrak{g} \mathfrak{t}(2)$ example appeared in a machine-learning scan of a subset of type IIA orientifolds on $T^{6} /\left(\mathbb{Z}_{2} \times \mathbb{Z}_{2}\right)$ with intersecting D6-branes [14].
}

[1] B. C. Allanach, B. Gripaios, and J. Tooby-Smith, Phys. Rev. Lett. 125, 161601 (2020).

[2] J. C. Pati and A. Salam, Phys. Rev. D 10, 275 (1974); 11, 703(E) (1975).

[3] H. Georgi and S. Glashow, Phys. Rev. Lett. 32, 438 (1974).

[4] H. Fritzsch and P. Minkowski, Ann. Phys. (N.Y.) 93, 193 (1975).

[5] H. Georgi, AIP Conf. Proc. 23, 575 (1975).

[6] See Supplemental Material at http://link.aps.org/ supplemental/10.1103/PhysRevD.104.035035 for the program output and additional information regarding the embeddings.

[7] A. Malcev, Izv. Akad. Nauk SSSR Ser. Mat. 8, 143 (1944), http://mi.mathnet.ru/eng/izv3724.

[8] E. B. Dynkin, Tr. Mosk. Mat. Obs. 1, 39 (1952), http://mi .mathnet.ru/eng/mmo3.

[9] E. B. Dynkin, Mat. Sb. 72, 349 (1952), http://mi.mathnet.ru/ eng/msb5435.
[10] M. Lorente and B. Gruber, J. Math. Phys. (N.Y.) 13, 1639 (1972).

[11] N. Yamatsu, arXiv:1511.08771.

[12] R. Feger and T. W. Kephart, Comput. Phys. Commun. 192, 166 (2015).

[13] R. Feger, T. W. Kephart, and R. J. Saskowski, Comput. Phys. Commun. 257, 107490 (2020).

[14] T. Li, A. Mansha, and R. Sun, arXiv:1912.11633.

[15] D. B. Costa, B. A. Dobrescu, and P. J. Fox, Phys. Rev. Lett. 123, 151601 (2019).

[16] B. C. Allanach, B. Gripaios, and J. Tooby-Smith, J. High Energy Phys. 05 (2020) 065.

[17] D. B. Costa, Phys. Rev. D 102, 115006 (2020).

[18] D. B. Costa, B. A. Dobrescu, and P. J. Fox, Phys. Rev. D 101, 095032 (2020).

[19] B. A. Dobrescu and P. J. Fox, arXiv:2012.04139. 